\title{
A unifying mode-coupling theory for transport properties of electrolyte solutions. II. Results for equal-sized ions electrolytes
}

Claudio Contreras Aburto, and Gerhard Nägele

Citation: The Journal of Chemical Physics 139, 134110 (2013); doi: 10.1063/1.4822298

View online: https://doi.org/10.1063/1.4822298

View Table of Contents: http://aip.scitation.org/toc/jcp/139/13

Published by the American Institute of Physics

\section{Articles you may be interested in}

A unifying mode-coupling theory for transport properties of electrolyte solutions. I. General scheme and limiting laws

The Journal of Chemical Physics 139, 134109 (2013); 10.1063/1.4822297

Mode coupling theory analysis of electrolyte solutions: Time dependent diffusion, intermediate scattering function, and ion solvation dynamics

The Journal of Chemical Physics 142, 124502 (2015); 10.1063/1.4915274

Frequency dependence of ionic conductivity of electrolyte solutions

The Journal of Chemical Physics 112, 1876 (2000); 10.1063/1.480751

Ion conductance in electrolyte solutions

The Journal of Chemical Physics 110, 10024 (1999); 10.1063/1.478876

Liquid-liquid phase transition in an ionic model of silica

The Journal of Chemical Physics 146, 234503 (2017); 10.1063/1.4984335

Long-time self-diffusion of charged spherical colloidal particles in parallel planar layers

The Journal of Chemical Physics 140, 244116 (2014); 10.1063/1.4884822

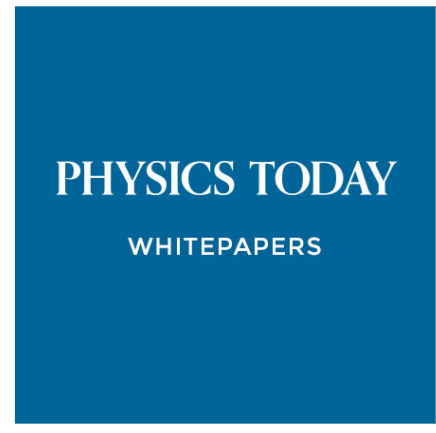

ADVANCED LIGHT CURE ADHESIVES

Take a closer look at what these environmentally friendly adhesive systems can do

\section{READ NOW}

PRESENTED BY Q MASTERBOND 


\title{
A unifying mode-coupling theory for transport properties of electrolyte solutions. II. Results for equal-sized ions electrolytes
}

\author{
Claudio Contreras Aburto ${ }^{a)}$ and Gerhard Nägele ${ }^{b)}$ \\ Institute of Complex Systems, ICS-3, Research Centre Jülich, D-52425 Jülich, Germany
}

(Received 23 May 2013; accepted 11 September 2013; published online 2 October 2013)

\begin{abstract}
On the basis of a versatile mode-coupling theory (MCT) method developed in Paper I [C. Contreras Aburto and G. Nägele, J. Chem. Phys. 139, 134109 (2013)], we investigate the concentration dependence of conduction-diffusion linear transport properties for a symmetric binary electrolyte solution. The ions are treated in this method as charged Brownian spheres, and the solventmediated ion-ion hydrodynamic interactions are accounted for also in the ion atmosphere relaxation effect. By means of a simplified solution scheme, convenient semi-analytic MCT expressions are derived for the electrophoretic mobilities, and the molar conductivity, of an electrolyte mixture with equal-sized ions. These expressions reduce to the classical Debye-Falkenhagen-Onsager-Fuoss results in the limit of very low ion concentration. The MCT expressions are numerically evaluated for a binary electrolyte, and compared to experimental data and results by another theoretical method. Our analysis encloses, in addition, the electrolyte viscosity. To analyze the dynamic influence of the hydration shell, the significance of mixed slip-stick hydrodynamic surface boundary conditions, and the effect of solvent permeability are explored. For the stick boundary condition employed in the hydrodynamic diffusivity tensors, our theoretical results for the molar conductivity and viscosity of an aqueous 1:1 electrolyte are in good overall agreement with reported experimental data for aqueous $\mathrm{NaCl}$ solutions, for concentrations extending even up to two molar. (C) 2013 AIP Publishing LLC. [http://dx.doi.org/10.1063/1.4822298]
\end{abstract}

\section{INTRODUCTION}

Electrolyte solutions play a central role in numerous electrochemical and biological processes, and in many industrial applications such as waste water treatment, chemical waste disposal, electrolysis, corrosion, and biofiltration. An issue of particular interest is the linear ion transport in strong electrolyte mixtures, which is the subject of ongoing experimental and theoretical research. ${ }^{1-3}$ The conduction-diffusion and rheological transport coefficients for low ion concentrations up to about $10^{-2} \mathrm{M}$ are well described by the DebyeFalkenhagen-Onsager-Fuoss (DFOF) limiting law expressions, based on the Debye-Hückel (DH) ion pair distribution functions (PDFs), with their characteristic square-root in concentration dependence. ${ }^{2-8}$ In more concentrated electrolyte systems, the concentration dependence of transport properties is determined not only by the electrostatic interactions, which are dominating at very low concentrations, but additionally by ion excluded volume effects and solvent-mediated ion-ion hydrodynamic interactions (HIs). The interplay of these generic interactions, which can be treated theoretically on the primitive model level using a continuum picture of the solvent, offers a severe theoretical challenge to the calculation of transport properties. In addition, ion-solvent specific effects on the molecular level caused by interactions between ions and surrounding solvent molecules may play a significant role. These

\footnotetext{
a) Present address: División de Ciencias e Ingenierías Campus León, Universidad de Guanajuato, 37150 León, Mexico.

b) Electronic mail: g.naegele@fz-juelich.de. Also at Department of Physics, Heinrich-Heine-University Düsseldorf, D-40225 Düsseldorf, Germany.
}

effects are most influential for the viscosity, and they have been addressed using concepts such as ion hydration, solvent polarization by an ion's electric field, and structure-breaking and structure-making properties of ions., ${ }^{2,3,9-11}$

Hence, there are analytic-theoretical methods on demand which allow for the computation of transport properties characterizing electrolyte conductivity, electrophoresis, diffusion, and viscosity in non-dilute solutions with all non-specific interactions included, in particular also the long-range HIs. These methods could help, e.g., to partially disentangle the ion-solvent specific effects on ion transport from the nonspecific ones.

Various routes have been followed in the past for calculating conduction-diffusion and rheological transport properties of non-dilute electrolyte solutions. These routes are mainly based on the primitive model $(\mathrm{PM})^{12,13}$ where the (hydrated) ions are treated as uniformly charged Brownian spheres embedded in a structureless Newtonian fluid characterized by the static dielectric constant $\epsilon$ and the shear viscosity $\eta_{0}$. The dynamics of the PM ions is commonly described by the many-particle Smoluchowski equation in conjunction with the Stokes equation of low-Reynolds-number solvent flow. ${ }^{2,14,15}$ Falkenhagen $^{3}$ and Ebeling et al. ${ }^{16,17}$ have extended the classical DFOF continuity equations approach to finite ion sizes. A considerable improvement over the classical DFOF theory was obtained by Bernard and co-workers in a series of publications ${ }^{18-23}$ where the DFOF approach has been combined with the analytic mean spherical approximation (MSA) solution ${ }^{24,25}$ for the ionic pair PDFs. The in general non-negligible influence of the HIs on the relaxation parts 
of the conduction-diffusion transport properties is disregarded in their treatment, except for the special case of self-diffusion. Moreover, the electrolyte viscosity has not been considered in their works.

In recent works, Chandra et al. have combined MCT with dynamic density functional theory (DDFT) arguments in deriving expressions for the molar conductivity and viscosity of strong electrolyte solutions. ${ }^{26-29}$ The excluded volume of the ions is incorporated in their hybrid method using Attard's analytic generalization of the Debye-Hückel PDFs. ${ }^{12}$ However, the influence of the HIs on the electrolyte viscosity, and on the relaxation effect contribution to the conductivity, has been disregarded in their MCT-DDFT treatment.

In the present paper, we present theoretical results for steady-state conduction-diffusion and rheological transport properties of binary electrolytes. These results are based on the PM, and the generalized Smoluchowski equation for the ion dynamics with the HIs accounted for. Our results are obtained using a simplified solution scheme of a versatile MCT method for linear transport properties of electrolyte mixtures, referred to as the simplified MCT-HIs scheme. For conduction-diffusion properties, this scheme has been developed in Paper II ${ }^{85}$ and for viscoelastic properties in a previous publication by the authors. ${ }^{30}$ In Paper II, ${ }^{85}$ analytic MCT expressions have been derived for pointlike ions with HIs included. These expressions reduce, for very low ion concentrations, to the corresponding DFOF limiting law results.

On the basis of the simplified MCT-HIs scheme, semianalytic MCT expressions for conduction-diffusion properties of a symmetric binary electrolyte are derived in the present work which apply also to non-dilute solutions. The simplified MCT-HIs expressions for the electrophoretic mobility, molar conductivity, self-diffusion, and sedimentation coefficients, and for the electrolyte viscosity are numerically evaluated using Attard's PDFs as the static input. The importance of HIs for the various transport properties is assessed from the comparison with corresponding results without HIs. Moreover, our theoretical results are compared with experimental data for the conductivity and viscosity of a 1:1 electrolyte, and with results obtained by the MCT-DDFT method of Chandra and Bagchi. ${ }^{27-29}$ To investigate the dynamic influence of the ion hydration shells on a coarse-grained, continuum level of description, the significance of mixed slip-stick hydrodynamic surface boundary conditions, and the effect of solvent permeability are explored (see Fig. 1). A consistently good agreement between reported conductivity and viscosity data for aqueous $\mathrm{NaCl}$ solutions and our MCT results is obtained, without any adjustable parameter, for stick boundary conditions employed for the hydrodynamic diffusivity tensors, and for concentrations extending even up to two molar.

The paper is structured as follows: Sec. II includes a discussion of the static PDFs input, and results for the hydrodynamic functions contributing to the linear transport properties of electrolyte solutions. Our results for the short-time and steady-state conduction-diffusion properties, as functions of the total ion concentration, are presented in Sec. III. The steady-state properties are calculated using semi-analytic expressions obtained from the simplified MCT-HIs treatment. The theoretical results for the conductivity are compared with

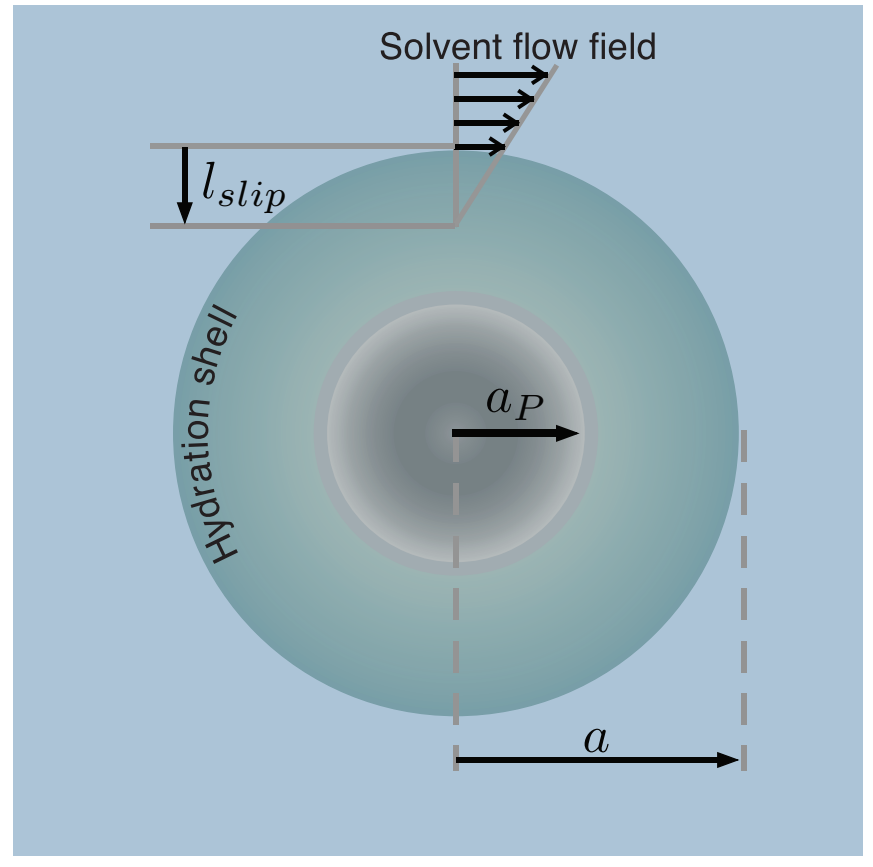

FIG. 1. Sketch of the PM-type ion-sphere model used in this work. Here, $a$ is the hydrodynamic radius of the hydrated ion, identified for simplicity with the excluded volume radius, and $l_{\text {slip }}$ is the hydrodynamic slip length appearing in the Navier mixed slip-stick hydrodynamic surface boundary condition (see Sec. IV). The hydrodynamic radius differs in general from the Pauling ionic radius, $a_{P}$, measured in crystallography experiments. For structure-making ions such as $\mathrm{Na}^{+}$in water, $a>a_{P}$ is observed.

those by Bagchi and Chandra. ${ }^{27-29}$ Moreover, the importance of HIs contributions to the relaxation parts of the transport coefficients is assessed. The results presented in Secs. II and III have been obtained using the stick hydrodynamic boundary condition (BC). The effects of mixed slip-stick BCs and possible solvent-permeability of ions on the electrolyte transport properties are analyzed in Sec. IV and in Appendix C. This analysis encloses also the electrolyte viscosity and sedimentation coefficient in the PM. Finally, Sec. V contains our conclusions.

To avoid repetitions, we will refer in the following to equations given already in Paper $\mathrm{I}^{85}$ using the format (I. . . ), where $\cdots$ stands for the referenced equation number in Paper I. ${ }^{85}$

\section{STATIC INPUT AND HYDRODYNAMIC INTERACTIONS}

In this section, we describe the analytic pair distribution functions used as input in the simplified MCT-HIs scheme calculations of electrolyte transport properties. Moreover, we specify the hydrodynamic diffusivity tensors employed in calculating the global hydrodynamic functions which contribute to the investigated short-time and steady-state conductiondiffusion and viscoelastic properties. Results for the global hydrodynamic functions are presented.

We treat strong electrolyte mixtures on the PM level where the hydrated electrolyte ions of components $\alpha=1, \ldots$, $m$ are described as uniformly charged hard spheres interacting statically by Coulomb and excluded volume forces. The 
solvent is modeled as a structureless continuum, characterized statically by the Bjerrum length $L_{B}=e^{2} /\left(\epsilon k_{B} T\right.$ ) (in Gaussian units), with proton charge $e$ and static dielectric constant $\epsilon$ at temperature $T$, and dynamically by the shear viscosity $\eta_{0}$. To allow for an analytic treatment, we assume that all ions of the electrolyte solution have the same excluded volume radius $a$ (diameter $\sigma=2 a$ ) taken equal to the hydrodynamic radius, and the same single-ion translational diffusion coefficient $D^{0}$. The ions of component $\alpha$ are characterized by the single-ion charge $z_{\alpha} e$, and the mean number concentration $n_{\alpha}$, so that

$$
n_{T}=\sum_{\gamma=1}^{m} n_{\gamma}
$$

is the total number density of ions in solution. Furthermore,

$$
\overline{z^{p}}=\sum_{\alpha=1}^{m} x_{\alpha} z_{\alpha}^{p},
$$

with $p \in\{1,2, \cdots\}$, is the $p$ th moment of the ion valency distribution, and $x_{\alpha}=n_{\alpha} / n_{T}$ is the molar fraction of $\alpha$-type ions. Total charge neutrality commands that $\bar{z}=0$.

\section{A. Static pair distribution functions input}

Let $c_{\alpha \beta}(q)$ denote the Fourier transform of the partial direct correlation function, $c_{\alpha \beta}(r)$, of two ions of components $\alpha$ and $\beta$, separated by the centre-to-centre distance $r$. In the linear MSA and Attard approximations for ions with equal excluded volume diameter $\sigma$, the symmetric $m \times m$ matrix, $\mathbf{C}(q)$ with elements $C_{\alpha \beta}(q)=\sqrt{n_{\alpha} n_{\beta}} c_{\alpha \beta}(q)$ can be orthogonally decomposed as

$$
\mathbf{C}(q)=n_{T} c_{H S}(q) \mathbf{P}_{n}+n_{T} \overline{z^{2}} c_{E L}(q) \mathbf{P},
$$

where $c_{H S}(q)$ is the Fourier transform of the direct correlation function, $c_{H S}(r)$, of neutral hard spheres of reduced particle concentration

$$
n_{T}^{*}=n_{T} \sigma^{3},
$$

and associated volume fraction $\phi_{T}=\pi n_{T}^{*} / 6$. The $m \times m$ matrices $\mathbf{P}$ and $\mathbf{P}_{n}$ with elements

$$
P_{\alpha \beta}=\frac{\left(x_{\alpha} x_{\beta}\right)^{1 / 2} z_{\alpha} z_{\beta}}{\overline{z^{2}}},
$$

and

$$
P_{n, \alpha \beta}=\left(x_{\alpha} x_{\beta}\right)^{1 / 2},
$$

are the projectors on the charge-density fluctuations and ion total number density fluctuations subspaces, respectively, which we have introduced already in Paper I. ${ }^{30,85}$

In the MSA, $c_{H S}(q)$ is given analytically by the PercusYevick (PY) solution for monodisperse hard spheres, ${ }^{31}$ whereas the electric direct correlation function part, $c_{E L}(q)$, is obtained from Fourier transforming the analytic solution for $c_{E l}(r)$ obtained by Waisman and Lebowitz. ${ }^{32}$ The analytic Attard expressions are discussed further down. In the pointion limit, both methods reduce to the DH result $c_{H S}(r)=0$ and $c_{E L}(r)=-L_{B} / r$, with corresponding Fourier transforms $c_{H S}(q)=0$ and $c_{E L}(q)=-4 \pi L_{B} / q^{2}$.
For an electrolyte consisting of ions with equal diameter $\sigma$, it is convenient to introduce the diameter-scaled dimensionless wavenumber $y=q \sigma$ and pair distance $x=r / \sigma$. The dimensionless, scaled direct correlation function constituents, $c_{Y}(y)$, defined by $n_{T}^{*} c_{Y}(y)=n_{T} c_{Y}(q)$ with $Y=\{H S, E L\}$, are related to their real-space dimensionless counterparts, $c_{Y}(x)$, by the one-dimensional Fourier-sine transform

$$
n_{T}^{*} c_{Y}(y)=n_{T}^{*} \frac{4 \pi}{y} \int_{0}^{\infty} d x x c_{Y}(x) \sin [y x] .
$$

Throughout this work we stick to the standard physics convention of using the same symbol for a function whose argument is expressed in different units, with the unit made visible by the employed argument symbol. The direct correlation functions matrix, $\mathbf{C}(y)$, considered as a function of reduced wavenumber $y$, is thus given by

$$
\mathbf{C}(y)=n_{T}^{*} c_{H S}(y) \mathbf{P}_{n}+n_{T}^{*} \overline{z^{2}} c_{E L}(y) \mathbf{P} .
$$

The associated static structure factor matrix, $\mathbf{S}(y)$, has the orthogonal decomposition

$$
\mathbf{S}(y)=(\mathbf{1}-\mathbf{C}(y))^{-1}=\mathbf{Q}+S_{H S}(y) \mathbf{P}_{n}+S_{E L}(y) \mathbf{P} .
$$

Here, $\mathbf{1}$ is the $m \times m$ unit matrix, and

$$
S_{H S}(y)=\frac{1}{1-n_{T}^{*} c_{H S}(y)}
$$

and

$$
S_{E L}(y)=\frac{1}{1-n_{T}^{*} \overline{z^{2}} c_{E L}(y)} .
$$

Moreover,

$$
\mathbf{Q}=\mathbf{1}-\mathbf{P}-\mathbf{P}_{n}
$$

and the three projection matrices $\left\{\mathbf{Q}, \mathbf{P}_{n}, \mathbf{P}\right\}$ are mutually orthonormal. In the DH point-ion limit, $S_{H S}(y)=1$ and

$$
S_{E L}(y)=\frac{y^{2}}{y^{2}+(\kappa \sigma)^{2}},
$$

with the Debye screening parameter, $\kappa$, given by

$$
\kappa^{2}=4 \pi L_{B} n_{T} \overline{z^{2}} .
$$

In the MSA and Attard approximations for equal-sized ions, the partial total correlation functions, $h_{\alpha \beta}(x)$, which are related to the $c_{\alpha \beta}(x)$ by the $m$-component Ornstein-Zernike equations, can be decomposed according to ${ }^{12,24,32}$

$$
h_{\alpha \beta}(x)=h_{H S}(x)+z_{\alpha} z_{\beta} h_{E L}(x),
$$

into pure hard-sphere and electro-steric parts. The real-space total correlation functions $h_{H S}(x)$ and $h_{E L}(x)$, which are used as static input in the calculation of the hydrodynamic functions, are numerically calculated by Fourier inversion of the analytically known right-hand sides of ${ }^{30}$

$$
h_{H S}(y)=c_{H S}(y) S_{H S}(y)
$$

and

$$
h_{E L}(y)=c_{E L}(y) S_{E L}(y) \text {, }
$$


respectively. In the Attard approximation, $h_{H S}(x)=-\theta(1-x)$ and

$$
h_{E L}(x)=-\frac{L_{B}}{\sigma} \frac{\Gamma_{r e n}}{x} \mathrm{e}^{-\kappa_{r e n} \sigma(x-1)} \theta(x-1),
$$

are both known analytically. Here,

$$
\kappa_{r e n}=\frac{\kappa}{\sqrt{1-(\kappa \sigma)^{2} / 2+(\kappa \sigma)^{3} / 6}}
$$

and

$$
\Gamma_{r e n}=\frac{\kappa_{r e n}^{2}}{\kappa^{2}\left(1+\kappa_{r e n} \sigma\right)}
$$

are renormalized screening and coupling parameters, respectively, determined such that the second moment condition is fulfilled by the Attard PDFs. ${ }^{12}$

We have determined earlier the viscosity of strong electrolyte solutions consisting of equal-sized, monovalent ions, from the simplified MCT-HIs method using both the MSA and the Attard ion PDFs as input. ${ }^{30}$ Although the PDFs predicted by the two methods are noticeably different from each other for $n_{T}>0.5 \mathrm{M}$, the corresponding viscosity results remain practically the same for substantially larger concentrations. Therefore, and for simplicity, only the Attard static input is used in the present paper. Moreover, transport properties results are presented only for solutions of monovalent ions of equal diameter (restricted PM). While the MSA and Attard PDFs inputs have been used in the literature also for divalent ions, ${ }^{22,29}$ it should be noted that for electrolyte solutions including non-monovalent ions, an improved static input is actually required, which properly accounts for ion pairing and nonlinear electrostatic screening tendencies.

\section{B. Hydrodynamic functions input}

In the following, we describe our treatment of the solvent-mediated ion-ion HIs. Additional information is contained in Appendix A.

Consider again an $m$-component PM system which has in total

$$
N=\sum_{\gamma=1}^{m} N_{\gamma}
$$

ions of equal hydrodynamic radius $a=\sigma / 2$ and equal singleion diffusion coefficient $D^{0}$, where $N_{\gamma}$ is the number of $\gamma$-type ions. The HIs between an ion $j \in \beta$ whose center is located at the position vector $\mathbf{R}_{j}^{\beta}$, and an ion $i \in \alpha$ at position $\mathbf{R}_{i}^{\alpha}$, are described by the $3 \times 3 N$-particle translational hydrodynamic diffusivity tensors, $\mathbf{D}_{i j}^{\alpha \beta}\left(\mathbf{R}^{N}\right)$, which depend on the $3 N$-dimensional configuration vector $\mathbf{R}^{N}$ of instantaneous ion positions. The tensor $\mathbf{D}_{i j}^{\alpha \beta}\left(\mathbf{R}^{N}\right) /\left(k_{B} T\right)$ linearly relates the hydrodynamic force on ion $j \in \beta$ to the resulting velocity change of ion $i \in \alpha$. It can be obtained, in principle, from solving the Stokes equation describing quasi-stationary creeping flow, with the hydrodynamic BCs specified on the surfaces of the $N$ ions. ${ }^{33,34}$ Since this hydrodynamic problem cannot be solved analytically, some approximations have to be made.

In the so-called pairwise-additivity (PA) approximation, the $\mathbf{D}_{i j}^{\alpha \beta}\left(\mathbf{R}^{N}\right)$ are approximated by the sum of two-sphere hydrodynamic mobilities according to ${ }^{14,35}$

$$
\begin{aligned}
\frac{\mathbf{D}_{i j}^{\alpha \beta}\left(\mathbf{R}^{N}\right)}{D^{0}}= & \delta_{i j}^{\alpha \beta}\left(\mathbf{1}+\sum_{\gamma=1}^{m} \sum_{l=1}^{N_{\gamma}}{ }^{\prime} \mathbf{a}_{11}\left(\mathbf{R}_{i}^{\alpha}-\mathbf{R}_{l}^{\gamma}\right)\right) \\
& +\left(1-\delta_{i j}^{\alpha \beta}\right) \mathbf{a}_{12}\left(\mathbf{R}_{i}^{\alpha}-\mathbf{R}_{j}^{\gamma}\right),
\end{aligned}
$$

where $\delta_{i j}^{\alpha \beta}$ is equal to one for $\alpha=\beta$ and $i=j$, and zero otherwise. The prime in the double sum indicates that the (divergent) self-terms $l=i$ and $\gamma=\alpha$ are excluded. Since all ions are assumed to be hydrodynamically identical, the hydrodynamic tensor mobility functions $\mathbf{a}_{11}$ and $\mathbf{a}_{12}$ are the same for all ion pairs. We have maintained here the component index notation for $\mathbf{D}_{i j}^{\alpha \beta}$, since ions of different components are statistically non-equivalent for different component valencies.

Because of the axial symmetry of the two-sphere problem, $\mathbf{a}_{11}$ and $\mathbf{a}_{12}$ can be decomposed into longitudinal and transverse components according to

$$
\mathbf{a}_{11}(\mathbf{r})=x_{11}(r) \widehat{\mathbf{r}} \widehat{\mathbf{r}}+y_{11}(r)(\mathbf{1}-\widehat{\mathbf{r}} \widehat{\mathbf{r}})
$$

and

$$
\mathbf{a}_{12}(\mathbf{r})=x_{12}(r) \widehat{\mathbf{r}} \widehat{\mathbf{r}}+y_{12}(r)(\mathbf{1}-\widehat{\mathbf{r}} \widehat{\mathbf{r}}),
$$

where $\widehat{\mathbf{r}}=\mathbf{r} / r$. Recursion relations for the series expansion of the isotropic functions $x_{i j}$ and $y_{i j}$ in powers of $1 / x=2 a / r$, and a lubrication correction for near-contact distances are provided by Jeffrey and Onishi. ${ }^{36}$ In our calculations of electrolyte transport coefficients invoking the PA approximation of the HIs, we use numerical tables for the $x_{i j}$ and $y_{i j}$ generated following the work of Jeffrey and Onishi. ${ }^{36}$

The near-field (NF) parts of the longitudinal and transversal functions $x_{i j}$ and $y_{i j}$, respectively, which are of $\mathcal{O}\left(1 / x^{4}\right)$ or shorter-ranged, are defined for stick hydrodynamic BCs as

$$
x_{i j}^{N F}=x_{i j}(x)-x_{\mathrm{ij}}^{R P}(x),
$$

with an analogous definition for the $y_{i j}^{N F}$. Here, $x_{11}^{R P}=0$, $y_{11}^{R P}=0$,

$$
x_{12}^{R P}=\frac{3}{4 x}-\frac{1}{8 x^{3}}
$$

and

$$
y_{12}^{R P}=\frac{3}{8 x}+\frac{1}{16 x^{3}} .
$$

The tensor functions $\mathbf{a}_{11}$ and $\mathbf{a}_{12}$, with $x_{i j}$ and $y_{i j}$ approximated by $x_{i j}^{R P}$ and $y_{i j}^{R P}$, respectively, constitute the wellknown Rotne-Prager (RP) approximation of the hydrodynamic diffusivity tensors, ${ }^{37}$ where only the $\mathcal{O}\left(1 / x^{3}\right)$ far-field part of the HIs is retained. The RP approximation has been used already in our simplified MCT-HIs calculations of the electrolyte viscosity, ${ }^{30}$ and it is employed in the present paper for most of the discussed results for conduction-diffusion properties. The changes introduced in the transport properties by using a RP-type approximation with mixed slip-stick or permeable-sphere BCs are discussed in Sec. IV, also in relation to colloidal systems.

On first sight, the easy-to-implement RP approximation appears to be cruder than the more elaborate PA approximation, since the latter accounts for the complete two-body HIs 
part, including lubrication. While for large colloidal particles lubrication is certainly important, its consideration becomes questionable for nano-sized (hydrated) ions which are not very much larger than individual solvent molecules. Moreover, and different from the PA approximation, the RP approximation has the important feature of preserving, for all physically allowed ion configurations, the positive definiteness of the exact $3 N \times 3 N$ hydrodynamic diffusivity matrix which has the exact $\mathbf{D}_{i j}^{\alpha \beta}$ as its entries, and of the associated hydrodynamic functions. Since the RP approximation is deducible from a minimal dissipation theorem, it can be used to obtain upper bounds on the exact short-time diffusion and sedimentation coefficients ${ }^{37,38}$ (see Sec. IV). We will compare our transport coefficient results with RP-HIs to those obtained using PA-HIs, to elucidate the effect of the near-field HIs contributions.

As we have explained in Paper I ${ }^{85}$ the effect of the HIs on the short-time dynamic correlations of the partial concentration fluctuations in an $m$-component PM system is described by the $m \times m$ hydrodynamic function matrix, $\mathbf{H}(q)$. According to Eq. (I.35) of Paper $\mathrm{I}^{85}$ the elements of this matrix can be decomposed into self- and distinct parts, which we rewrite here as

$$
H_{\alpha \beta}(q)=\delta_{\alpha \beta} D_{\alpha}^{S}+H_{\alpha \beta}^{d}(q) .
$$

Here, $D_{\alpha}^{S}$ is the short-time self-diffusion coefficient of $\alpha$-type ions, and $H_{\alpha \beta}^{d}(q)$ is the distinct hydrodynamic function part, defined in Eqs. (I.36) and (I.37), respectively. The distinct part depends on the modulus, $q$, of the scattering wavenumber $\mathbf{q}$ with associated unit vector $\hat{\mathbf{q}}=\mathbf{q} / q$. Without HIs, $\mathbf{H}$ becomes a diagonal matrix with $D^{0}$ as its diagonal entries.

For the MSA and Attard static inputs of a $m$ component system of equal-size ions, total electroneutrality implies that the short-time self-diffusion coefficients, $D_{\alpha}^{S}$, are all equal to the short-time self-diffusion coefficient, $D_{H S}^{S}$, of a monodisperse system of neutral hard spheres with total volume fraction $\phi_{T}$, independent of the component index. Moreover, on the PA and MSA/Attard levels of approximation, the hydrodynamic matrix function decouples into steric and electro-steric parts according to

$$
\mathbf{H}(y)=D^{S} \mathbf{Q}+H_{N N}(y) \mathbf{P}_{n}+\frac{1}{\overline{z^{2}}} H_{Z Z}(y) \mathbf{P} .
$$

Explicit expressions for the short-time self-diffusion coefficient $D^{S}$, and the so-called number-number $(N N)$ and charge-charge $(Z Z)$ global hydrodynamic functions $H_{N N}(y)$ and $H_{Z Z}(y)$, respectively, are derived in Appendix A. Expressions are presented both on the RP hydrodynamic far-field level, and in addition for the situation where two-body nearfield HIs are fully accounted for (PA approximation). Global hydrodynamic functions on the RP level only have been discussed before ${ }^{30}$ in relation to the electrolyte viscosity.

In the simplified MCT-HIs based numerical results for symmetric 1:1 electrolytes presented in this paper, the value of the mean ion diameter has been taken as $\sigma=a_{+}+a_{-}$, where $a_{+}$and $a_{-}$are the hydrodynamic radii of the cationic and anionic particle species, respectively. The values of $a_{+}$ and $a_{-}$are determined from applying the Stokes-Einstein relation, with perfect slip $\mathrm{BC}$, to the reported experimental
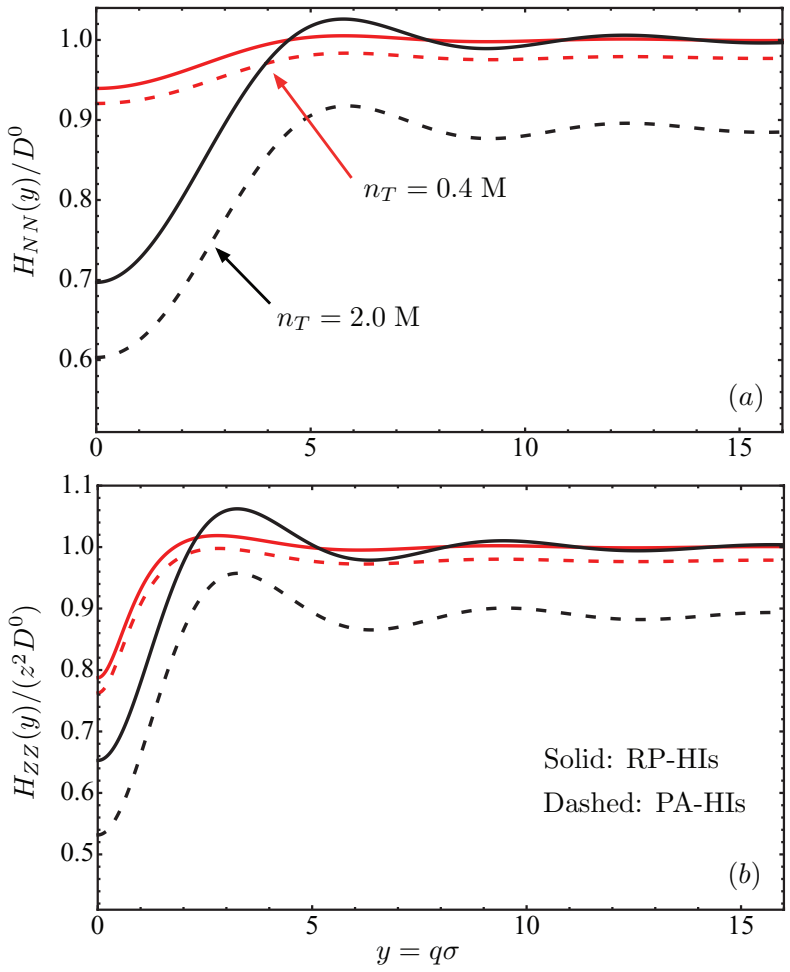

FIG. 2. Non-dimensionalized global hydrodynamic functions $H_{N N}(y)$ (a) and $H_{Z Z}(y)$ (b) of an aqueous symmetric 1:1 electrolyte (where $\overline{z^{2}}=1$ ) with ion diameter $\sigma=4.58 \AA$, and Bjerrum length $L_{B}=7.1 \AA$, characteristic of water at room temperature. The total ion concentrations considered are $n_{T}=0.4$ $\mathrm{M}$ (red curves) and $n_{T}=2 \mathrm{M}$ (black curves). Dashed lines are results for the HIs treated on the pairwise additive level using numerically precise two-body hydrodynamic mobility tensors (PA approximation). Solid lines are results obtained using the far-field RP-HIs approximation. Throughout this work, the Attard ion pair distribution functions are used as the static input.

values for the self-diffusion coefficients of cations, $D_{+}^{0}$, and anions, $D_{-}^{0}$, both measured for very low concentrations. To be specific, in our numerical study of conduction-diffusion and viscosity properties system parameters are used for aqueous $\mathrm{NaCl}$ electrolyte solutions at room temperature. An extended comparison with experimental data for other electrolytes will be made in a separate communication, where in addition the validity of the Walden rule and of generalized Stokes-Einstein and Nernst-Einstein relations is explored using the MCT-HIs method. For $\mathrm{Na}^{+}$and $\mathrm{Cl}^{-}$ions in water at $25^{\circ} \mathrm{C}$, where $\eta_{0}=0.89 \mathrm{cP}$, the experimental values are $D_{+}^{0}=1.33 \times 10^{-5} \mathrm{~cm}^{2} / \mathrm{s}$ and $D_{-}^{0}=2.03 \times 10^{-5} \mathrm{~cm}^{2} / \mathrm{s}$, leading with $a_{+}=2.77 \AA$ and $a_{-}=1.81 \AA$ to $\sigma=4.58 \AA$ for the mean ion diameter. ${ }^{43}$ This diameter value is used as input in most of the numerical results presented below.

Figure 2 displays results for the global hydrodynamic functions $H_{N N}(y)$ and $H_{Z Z}(y)$, respectively, calculated according to Eqs. (A14) and (A15) with the HIs treated on the PA and RP levels of approximation. In the RP approximation, the NF contributions are disregarded. Two different values of the total concentration, namely $n_{T}=0.4 \mathrm{M}$ (red curves) and $n_{T}$ $=2 \mathrm{M}$ (black curves) are considered. The inclusion of nearfield two-body HIs leads to a downshift of the hydrodynamic function curves which is more pronounced for larger ion concentrations. Even for the largest considered concentration of 
$2 \mathrm{M}$, the total ionic volume fraction, $\phi_{T} \approx 0.06$, is small enough that three-body and higher-order HIs contributions are comparatively small. On the RP level of HIs, the reduced hydrodynamic functions depicted in Fig. 2 asymptote to the value one at large wavenumbers, for the reason that $D_{\alpha}^{S}$ is approximated, decently well actually for the considered ion concentrations, by its infinite dilution value $D_{\alpha}^{0}=D^{0}$. Observe here the different scales in the ordinate axes of Figs. 2(a) and 2(b).

\section{CONDUCTION-DIFFUSION TRANSPORT COEFFICIENTS}

In this section, we derive simplified MCT-HIs expressions for short-time and steady-state conduction-diffusion properties of electrolyte mixtures consisting of equal-sized ions. The ion-ion HIs are treated both on the RP and pairwiseadditive (PA) levels of approximation. Explicit numerical results are presented for an aqueous 1:1 electrolyte, and they are compared with experimental data, and with the predictions by the MCT-DDFT approach of Chandra et al. ${ }^{26-29}$

\section{A. Partial mobilities}

We start by summarizing salient expressions for the steady-state partial mobilities derived in Paper I. ${ }^{85}$ These expressions are used here in our simplified MCT-HIs calculations of transport coefficients. The conduction-diffusion transport properties are obtained as linear combinations of steady-state partial mobilities, $\mu_{\alpha \beta}^{L}$, forming the elements of the symmetric and positive definite $m \times m$ mobility matrix, $\boldsymbol{\mu}^{L}$, of a general $m$-component PM electrolyte. The partial mobilities are the sum,

$$
\mu_{\alpha \beta}^{L}=\mu_{\alpha \beta}^{S}+\Delta \mu_{\alpha \beta},
$$

of a short-time linear response part given by

$$
k_{B} T \mu_{\alpha \beta}^{S}=\lim _{q \rightarrow 0} H_{\alpha \beta}(q) \equiv H_{\alpha \beta}
$$

and the relaxation part (see Paper $\mathrm{I}^{44,85}$ ),

$$
k_{B} T \Delta \mu_{\alpha \beta}=-\left(\left[\mathbf{1}+\mathbf{m}^{c, i r r} \cdot \mathbf{H}^{-1}\right]^{-1} \cdot \mathbf{m}^{c, i r r}\right)_{\alpha \beta},
$$

where $\mathbf{H}$ is the $m \times m$ matrix with the zero- $q$ limiting partial hydrodynamic functions, $H_{\alpha \beta}$, as its elements. Moreover,

$$
\mathbf{m}^{c, i r r}=\lim _{q \rightarrow 0} \int_{0}^{\infty} d t \mathbf{M}^{c, i r r}(q, t)
$$

is the zero wavenumber limit of the time-integrated (irreducible) collective memory function matrix, $\mathbf{M}^{\text {c,irr }}(q, t)$, introduced in Paper I. ${ }^{85}$ In the simplified MCT-HIs approach, the time-integrated collective memory function matrix is approximated by (see Paper $\mathrm{I}^{85}$ for details)

$$
\begin{aligned}
m_{\alpha \beta}^{c, i r r}= & \frac{D_{\alpha}^{S} D_{\beta}^{S}}{6 \pi^{2}\left(n_{\alpha} n_{\beta}\right)^{1 / 2}} \int_{0}^{\infty} d t \\
& \times \int_{0}^{\infty} d k k^{4}\left\{F_{\alpha \beta}(k, t)(\mathbf{C}(k) \cdot \mathbf{F}(k, t) \cdot \mathbf{C}(k))_{\alpha \beta}\right. \\
& \left.-(\mathbf{C}(k) \cdot \mathbf{F}(k, t))_{\alpha \beta}(\mathbf{F}(k, t) \cdot \mathbf{C}(k))_{\alpha \beta}\right\} .
\end{aligned}
$$

Here, $\mathbf{F}(k, t)$ is the symmetric $m \times m$ matrix of equilibrium partial dynamics structure factors, $F_{\alpha \beta}(k, t)$, depending on the correlation time $t$ and the wavenumber $k$, and $D_{\alpha}^{S}$ is the short-time self-diffusion coefficient of $\alpha$-type ions. Equation (34) generalizes the simplified MCT-HIs expression for $m_{\alpha \beta}^{c, i r r}$ given in Paper $\mathrm{I}^{85}$ to the situation where, in addition to the RP far-field HIs, also near-field hydrodynamic contributions to $\mathbf{F}(k, t)$ are accounted for. The latter lead to $D_{\alpha}^{S}<D_{\alpha}^{0}$.

As explained in Paper I ${ }^{85}$ (Eqs. (I.13) and (I.14)), the steady-state partial mobilities are given by the sum,

$$
\mu_{\alpha \beta}^{L}=\delta_{\alpha \beta} \frac{D_{\alpha}^{L}}{k_{B} T}+\left(\mu_{\alpha \beta}^{L}\right)^{\text {cross }},
$$

of a diagonal self-part proportional to the long-time selfdiffusion coefficient, $D_{\alpha}^{L}$, which quantifies the long-time slope of the mean-squared displacement of $\alpha$-type ions, and a non-diagonal cross part, $\left(\mu_{\alpha \beta}^{L}\right)^{\text {cross }}$, whose diagonal matrix elements are in general non-zero.

The electrophoretic mobility, $\mu_{\alpha}^{e l}$, of $\alpha$-type ions in a weak external electric field can be expressed in terms of the above partial mobilities as (see Eq. (I.15) in Paper $\mathrm{I}^{85}$ )

$$
\mu_{\alpha}^{e l}=\frac{z_{\alpha} e}{k_{B} T} D_{\alpha}^{L}+\sum_{\gamma=1}^{m}\left(\frac{n_{\gamma}}{n_{\alpha}}\right)^{1 / 2} z_{\gamma} e\left(\mu_{\alpha \gamma}^{L}\right)^{\text {cross }},
$$

where the sum extends over all $m$ ion components.

\section{B. Short-time transport properties}

We consider first the conduction-diffusion properties in the short-time regime, where changes in the ion configuration are very small. The $m \times m$ short-time mobility matrix part, $\boldsymbol{\mu}^{S}$, for equal-sized ions and based on the MSA or Attard pair correlation functions input, is readily deduced from Eq. (29) as

$$
k_{B} T \boldsymbol{\mu}^{S}=D^{S} \mathbf{Q}+H_{N N} \mathbf{P}_{n}+\frac{1}{\overline{z^{2}}} H_{Z Z} \mathbf{P},
$$

where $D^{S}=D_{H S}^{S}$. Here, $H_{N N}$ and $H_{Z Z}$ are short-hand notations for the zero- $y$ limits of $H_{N N}(y)$ and $H_{Z Z}(y)$, respectively. The more demanding calculation of the relaxation mobility matrix part, $\Delta \boldsymbol{\mu}$, required as the second ingredient to the steady-state mobility matrix, is addressed in Subsection III C.

Note that to the matrix $\boldsymbol{\mu}^{S}$ contribute, first, the shorttime self-diffusion coefficient $D_{H S}^{S}$, second the (short-time) sedimentation coefficient $K_{H S}^{\text {sed }}$ of a suspension of equal-sized neutral hard spheres (see Appendix C), related to $H_{N N}$ by ${ }^{40}$

$$
K_{H S}^{\text {sed }}=\frac{H_{N N}}{D^{0}},
$$

and third the reduced short-time electrophoretic mobility of $\alpha$-type ions, $\mu_{\alpha}^{e l, S}$. The third contribution for an arbitrary $m$ component electrolyte is related to $H_{Z Z}$ by

$$
\mu_{\alpha}^{e l, S}=\frac{e}{k_{B} T} \frac{\overline{z^{2}}}{x_{\alpha} z_{\alpha}}[\mathbf{P} \cdot \mathbf{H}(0)]_{\alpha \alpha},
$$

which restates Eq. (I.38) for $\mu_{\alpha}^{e l, S}$ in terms of the projector matrix $\mathbf{P}$ defined in Eq. (5). 
For equal-sized ions with equal single-ion diffusion coefficient $D^{0}$, and pair correlations treated on the linear MSA or Attard level, Eq. (29) applies, leading in combination with Eq. (39) to

$$
\frac{\mu_{\alpha}^{e l, S}}{\mu_{\alpha}^{e l, 0}}=\frac{H_{Z Z}}{\overline{z^{2}} D^{0}}
$$

for the ratio of short-time and infinite dilution electrophoretic mobilities. The electrophoretic mobility of $\alpha$-type ions at infinite dilution, as defined in Eq. (I.9), is $\mu_{\alpha}^{e l, 0}=z_{\alpha} e D^{0} /\left(k_{B} T\right)$. Notice that the mobility ratio is independent of the component index $\alpha$ for the equal-sized ions.

In the DFOF regime of low concentrations $\left(n_{T} \lesssim 0.01 \mathrm{M}\right)$, where monovalent ions are describable by the DH pair correlation functions of pointlike ions, the short-time electrophoretic mobilities in Eq. (40) reduce to the limiting law result,

$$
\mu_{\alpha}^{e l, S}=\mu_{\alpha}^{e l, 0}-\frac{z_{\alpha} e}{k_{B} T} D_{\kappa}=\frac{z_{\alpha} e}{k_{B} T}\left(D^{0}-D_{\kappa}\right),
$$

which has the characteristic square-root in $n_{T}$ (i.e., linear in $\kappa$ ) dependence. ${ }^{5}$ Here, $D_{\kappa}=k_{B} T \kappa /\left(6 \pi \eta_{0}\right)$ introduced already in Eq. (I.84), is a self-diffusion coefficient associated with a spherical body of hydrodynamic radius equal to the Debye length $1 / \kappa$. In performing the zero ion-radius limit $a \rightarrow 0$ in Eq. (40), the single-ion diffusion coefficient $D^{0}$ is treated as an independent and experimentally given quantity. Note that $D_{\kappa} \ll D^{0}$ applies in the low concentration regime where the DFOF limiting law expressions are applicable. ${ }^{30}$

Figure 3 shows the short-time conduction-diffusion properties $D^{S} / D^{0}, K_{H S}^{\text {sed }}$ and $\mu^{e l, S} / \mu^{e l, 0}$, of an aqueous symmetric 1:1 electrolyte, as functions of the total ion number concentration $n_{T}$ (lower horizontal scale) and total ion volume fraction $\phi_{T}$ (upper scale). The Attard ionic PDFs are used as static input in Eqs. (A14) and (A15). This is justifiable in the considered range $\phi_{T} \lesssim 0.06$ of small ion volume fractions. The HIs

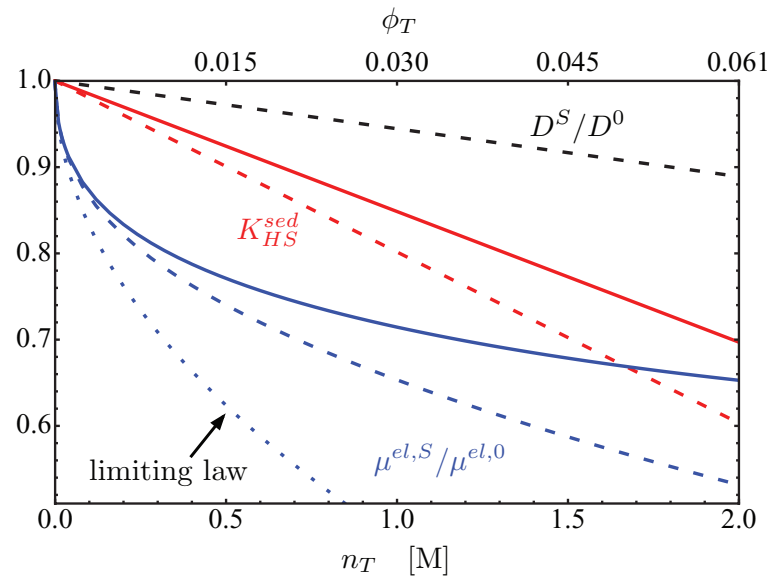

FIG. 3. Calculated normalized short-time conduction-diffusion transport coefficients (as indicated) of an aqueous symmetric 1:1 electrolyte (where $\overline{z^{2}}=1$ ) for system parameters as in Fig. 2. Solid lines are results based on HIs treated on the far-field RP-HIs level. Dashed lines are results obtained using the PA approximation. The upper horizontal scale is for the total ion volume fraction $\phi_{T}$. Dotted blue line: DFOF limiting law result for $\mu_{\alpha}^{e l, S}$ according to Eq. (41), normalized by $\mu_{\alpha}^{e l, 0}$. Note that the ratio $\mu_{\alpha}^{e l, S} / \mu_{\alpha}^{e l, 0}$ is the same for cations and anions. are treated on the PA and far-field RP levels of approximation, respectively. The slowing influence of the near-field two-body part of the HIs included in the PA approximation leads to a reduction of all three considered short-time transport properties. In particular, the short-time ion self-diffusion coefficient, $D^{S}=D_{H S}^{S}$, of cations and anions in a $2 \mathrm{M}$ solution is reduced in magnitude by about $10 \%$ below its infinite dilution value $D^{0}$.

At large concentrations, the PA approximation has the tendency to overestimate the decrease in the reduced shorttime conduction-diffusion transport coefficients, which can be attributed to the neglected three-body and higher-order nearfield HIs contributions. These contributions cause a weakening of the strength (but not of the range) of the HIs. Figure 3 illustrates for non-dilute electrolytes a pronounced deviation of the common normalized short-time electrophoretic mobility of cations and anions from the DFOF limiting law result. The latter applies only if $n_{T} \lesssim 0.01 \mathrm{M}$.

\section{Simplified MCT treatment with HIs}

As discussed in Sec. IV C of Paper I, ${ }^{85}$ the simplified mode-coupling theory method with hydrodynamic interactions included (MCT-HIs) consists of using the short-time approximation, $\mathbf{F}^{S}(q, t)$, of the dynamic structure factor matrix $\mathbf{F}(q, t)$ as input in the MCT expression for the time-integrated collective memory function matrix $\mathbf{m}^{c, i r r}$ in Eq. (34). This approximation is reasonably good for moderately concentrated electrolyte solutions, and it leads to great analytic progress. A related simplified MCT-HIs approach succeeded in the calculation of the electrolyte viscosity, and the associated shear relaxation function. ${ }^{30}$

In Smoluchowski dynamics, the short-time dynamic structure factor matrix of an $m$-component electrolyte reads

$$
\mathbf{F}^{S}(q, t)=\mathrm{e}^{-q^{2} \mathbf{H}(q) \cdot \mathbf{S}^{-1}(q) t} \cdot \mathbf{S}(q) .
$$

This matrix can be written as the sum of $m$ exponentially decaying normal modes where one mode, the so-called Debye mode, is non-diffusive, meaning that its relaxation rate remains non-zero in the limit $q \rightarrow 0$. For equal-sized ions and when the Attard or MSA PDFs are used as input, there are only three different relaxation rates. Insertion of the orthogonal decompositions of $\mathbf{H}(y)$ according to Eq. (A11), and of $\mathbf{S}(y)$ according to Eq. (9), into Eq. (42) leads to the normal mode expansion result

$$
\begin{aligned}
\mathbf{F}^{S}(y, \tau)= & \mathrm{e}^{-\lambda_{\text {self }}(y) \tau} \mathbf{Q}+\mathrm{e}^{-\lambda_{H S}(y) \tau} S_{H S}(y) \mathbf{P}_{n} \\
& +\mathrm{e}^{-\lambda_{E L}(y) \tau} S_{E L}(y) \mathbf{P},
\end{aligned}
$$

for $\mathbf{F}^{S}(q, t)$, expressed in terms of the reduced variables $y$ and $\tau$. We have introduced here the reduced correlation time

$$
\tau=t / \tau_{\sigma},
$$

where

$$
\tau_{\sigma}=\frac{\sigma^{2}}{D^{S}}
$$

is the characteristic self-diffusion time over the distance $\sigma$. 
The diffusive mode reduced relaxation rates are

$$
\lambda_{\text {self }}(y)=y^{2},
$$

and

$$
\lambda_{H S}(y)=y^{2} \frac{1}{D^{S}} \frac{H_{N N}(y)}{S_{N N}(y)} .
$$

The Debye mode relaxation rate is

$$
\lambda_{E L}(y)=y^{2} \frac{1}{D^{S}} \frac{H_{Z Z}(y)}{S_{Z Z}(y)},
$$

where $D^{S}=D_{H S}^{S}$. Here, $S_{N N}(y)=\operatorname{Tr}\left[\mathbf{P}_{n} \cdot \mathbf{S}(y)\right]=S_{H S}(y)$ is the number-number static structure factor associated with fluctuations in the total number density, and $S_{Z Z}(y)$ $=\overline{z^{2}} \operatorname{Tr}[\mathbf{P} \cdot \mathbf{S}(y)]=\overline{z^{2}} S_{E L}(y)$ is the static structure factor associated with fluctuations in the total charge density around its mean value zero. Notice that $\lambda_{E L}(y \rightarrow 0)>0$, since $S_{Z Z}(y)$ $=y^{2} \bar{z}^{2} /(\kappa \sigma)^{2}+\mathcal{O}\left(y^{4}\right)$. The mode with relaxation rate $\lambda_{\text {self }}$ is associated with the self-diffusion coefficient $D^{S}$.

In the point-ion limit $\sigma \rightarrow 0$, it follows with $D^{S} \rightarrow D^{0}$ that the normal mode associated with the relaxation rate $\lambda_{H S}$ becomes part of the degenerate subspace of normal modes characterized by $\lambda_{\text {self }}(y)$, and that $\lambda_{E L}(y) \tau \rightarrow \lambda_{D}(q) t$. The expression for the Debye frequency, $\lambda_{D}$, of hydrodynamically interacting point ions is given in Eq. (I.95).

\section{Self-diffusion mobility part}

We analyze here the long-time self-diffusion coefficient, $D_{\alpha}^{L}$, quantifying the long-time slope of the mean-squared displacement of $\alpha$-type ions. According to Eq. (36), $D_{\alpha}^{L}$ contributes as the self-part to the electrophoretic mobility of $\alpha$-type ions. In the MCT-HIs approach based on the collective memory function matrix, $D_{\alpha}^{L}$ is determined from mentally singling out a few $\alpha$-type ions to form a fictitious additional component $\alpha^{*}$, taking subsequently the limit $n_{\alpha}^{*} \rightarrow 0$. As a consistency check, we determine here $D_{\alpha}^{L}$ alternatively using the MCT expression for the self-intermediate scattering function of $\alpha$-type ions. In this self-dynamics approach, $D_{\alpha}^{L}$ can be calculated starting from the exact relation ${ }^{45}$ (see Paper $\mathrm{I}^{85}$ )

$$
\frac{D_{\alpha}^{L}}{D^{S}}=\left(1+\frac{\triangle \zeta_{\alpha}}{\zeta^{S}}\right)^{-1}
$$

between $D_{\alpha}^{L}$ and the self-correlation friction coefficient $\Delta \zeta_{\alpha}$. The latter coefficient quantifies the electrolyte friction contribution originating from the non-instantaneous relaxation of the ion atmosphere formed around each $\alpha$-type ion. It is equal to the time-integrated (irreducible) self-memory function evaluated in the limit $q \rightarrow 0 .{ }^{45}$ Note that $D_{\alpha}^{S}$, and its associated short-time friction coefficient $\zeta_{\alpha}^{S}=k_{B} T / D_{\alpha}^{S}$ appearing in Eq. (49), depend in general on the ion component index $\alpha$. However, for an electrolyte mixture of equal-sized ions with static pair correlations described on the MSA or Attard level, $D_{\alpha}^{S}=D^{S}=D_{H S}^{S}$ is independent of $\alpha$ (see Appendix A), and in consequence also $\zeta_{\alpha}^{S}=\zeta^{S}=k_{B} T / D_{H S}^{S}$.

The substitution of $\mathbf{F}^{S}(q, t)$ as an approximation for $\mathbf{F}(q, t)$ into the MCT expression for $\Delta \zeta_{\alpha}$ given in Eq. (I.133), and with $D_{\alpha}^{0}$ replaced by $D^{S}$, leads to the analytic simplified
MCT-HIs result,

$$
\frac{\Delta \zeta_{\alpha}}{\zeta^{S}}=\frac{\Delta \zeta_{H S}}{\zeta^{S}}+z_{\alpha}^{2} \frac{\Delta \zeta_{E L}}{\zeta^{S}},
$$

for the relaxational self-friction coefficient. The coefficient consists of a pure hard-sphere part,

$$
\frac{\Delta \zeta_{H S}}{\zeta^{S}}=\frac{\phi_{T}}{\pi^{3}} \int_{0}^{\infty} d y y^{2} \frac{\left(h_{H S}(y)\right)^{2}}{S_{N N}(y)+H_{N N}(y) / D^{S}}
$$

and an electro-steric part

$$
\frac{\Delta \zeta_{E L}}{\zeta^{S}}=\frac{\phi_{T}}{\pi^{3}} \overline{z^{2}} \int_{0}^{\infty} d y y^{2} \frac{\left(h_{E L}(y)\right)^{2}}{S_{E L}(y)+H_{Z Z}(y) /\left(\overline{z^{2}} D^{S}\right)} .
$$

In the limit $\sigma \rightarrow 0$ for which $h_{H S}(y) \rightarrow 0$ and $\zeta^{S}$ $\rightarrow \zeta^{0}=k_{B} T / D^{0}$, only the electrostatic contribution to the relaxational self-friction coefficient remains, giving rise to the limiting law result for $\lim _{\sigma \rightarrow 0} \Delta \zeta_{\alpha} / \zeta^{S}$ included in Eq. (I.135). In this point-ion limit, and to linear order in $\kappa$, we obtain the DFOF limiting law result, ${ }^{7}$

$$
\frac{D_{\alpha}^{L}}{D^{0}}=1-z_{\alpha}^{2} f^{p} \kappa L_{B},
$$

for the long-time self-diffusion coefficient, $D_{\alpha}^{L}$, of $\alpha$-type ions in a dilute solution of equal-sized ions with single-ion diffusion coefficient $D^{0}$. Here, $f^{p}=(2-\sqrt{2}) / 6$ is a numerical factor characteristic of the electrostatic interactions.

Figure 4 displays our simplified MCT-HIs results for the relaxational self-friction coefficient, $\Delta \zeta$, of a symmetric aqueous 1:1 electrolyte together with its hard-sphere and electro-steric constituents, as functions of the total ion concentration (lower horizontal scale), and the reduced Debye screening parameter (upper horizontal scale). Since $\left|z_{c}\right|$ $=\left|z_{a}\right|$, the long-time self-diffusion coefficient $D_{\alpha}^{L}=D^{L}$ is for this symmetric situation the same for cations and anions. Note further that for $n_{T} \gtrsim 1 \mathrm{M}$, the screening length becomes

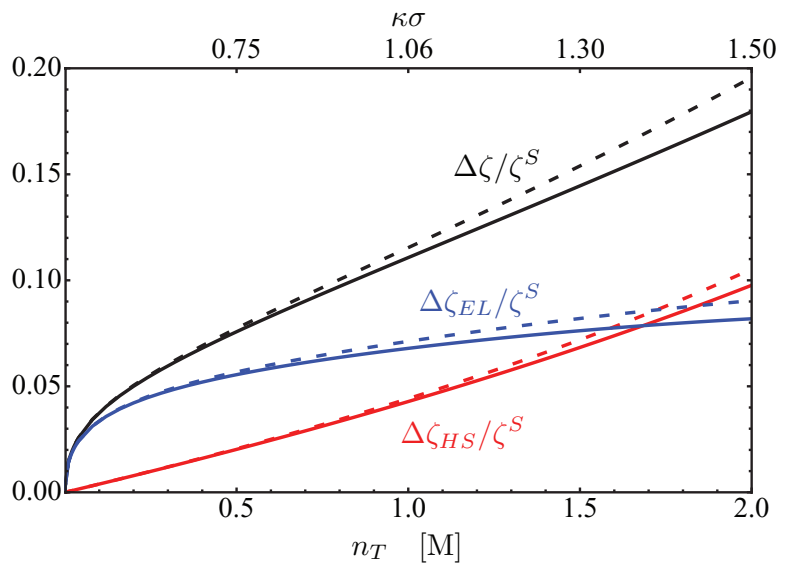

FIG. 4. Simplified MCT-HIs results for the reduced relaxational self-friction coefficient, $\Delta \zeta / \zeta^{S}$, and its hard-sphere (HS) and electro-steric (EL) parts, of an aqueous 1:1 electrolyte with parameters as in Fig. 2. Here, $\zeta^{S}=k_{B} T / D^{S}$ is the short-time self-friction coefficient of monovalent anions and cations. Solid lines: Results based on HIs treated on the RP far-field level. Dashed lines: Results based on HIs treated in PA approximation. Consistent with the limiting law result for self-diffusion, the electro-steric self-friction contribution $\Delta \zeta_{E L}$ dominates at small ion concentrations. Upper horizontal scale: Ratio, $\kappa \sigma$, of mean ion diameter $\sigma$ and Debye screening length $1 / \kappa$. 


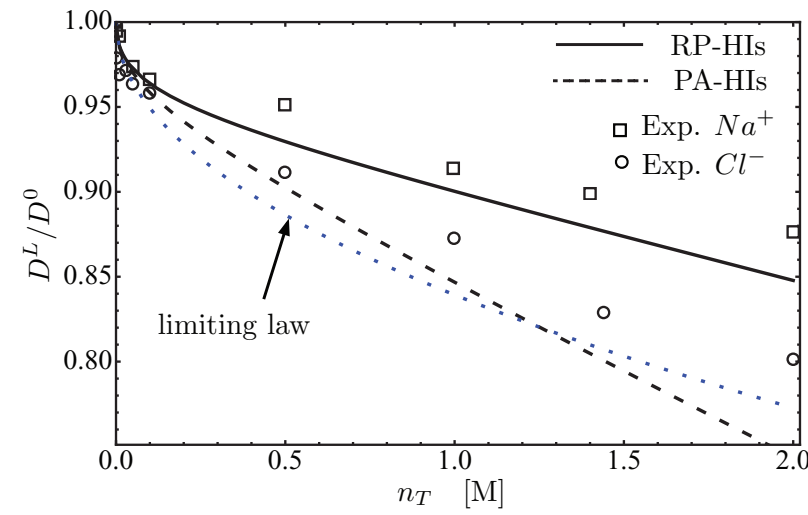

FIG. 5. Long-time self-diffusion coefficient, $D^{L}$, of a symmetric aqueous 1:1 electrolyte with parameters as in Fig. 2, normalized by its zero concentration value $D^{0}$, and plotted as a function of the total electrolyte concentration. Solid black line: Simplified MCT result obtained using the RP-HIs approximation both in $\Delta \zeta_{\alpha}$ and $\zeta^{S}$. Dashed black line: Corresponding result using PA-HIs in $\Delta \zeta_{\alpha}$ and $\zeta^{S}$. The theoretical predictions are compared with the experimental (reduced) self-diffusion coefficient, $D_{\mathrm{Na}^{+}}^{L} / D_{\mathrm{Na}^{+}}^{0}$, of sodium ions (open squares) and the (reduced) self-diffusion coefficient, $\mathrm{D}_{\mathrm{Cl}^{-}}^{L} / D_{\mathrm{Cl}^{-}}^{0}$, of chloride ions (open circles) measured in water at $25^{\circ} \mathrm{C} .{ }^{46}$ Blue dotted line: DFOF limiting law result according to Eq. (53).

smaller than the ion diameter. The electro-steric friction contribution, $\Delta \zeta_{E L}$, which at very low ion concentration increases proportional to $\sqrt{n_{T}}$, dominates the hard-sphere part $\Delta \zeta_{H S}$ for smaller concentrations, consistent with the limiting law transport coefficients behavior of strong electrolytes. The ratio, $\Delta \zeta / \zeta^{S}$, of relaxation and short-time friction coefficients, and in consequence also the ratio, $D^{L} / D^{S}$, of long-time and short-time self-diffusion coefficients of cations and anions, is practically determined by the far-field HIs part only. There is just a slight increase of $\Delta \zeta / \zeta^{S}$ (decrease of $D^{L} / D^{S}$ ) caused by the near-field HIs contributions, visible in the figure at larger concentrations only. In RP approximation, $D^{S}$ is approximated by its infinite dilution value $D^{0}$, whereas $D^{S}<D^{0}$ is obtained in PA approximation (see also Fig. 3).

In Fig. 5, theoretical results are shown for the normalized long-time self-diffusion coefficient, $D^{L}$, of equal-sized cations and anions of diameter $\sigma=4.58 \AA$ in a symmetric (monovalent) electrolyte. These results have been obtained using Eq. (A9) for $D^{S}$, the simplified MCT-HIs expression for $\Delta \zeta$ in Eq. (50), and Attard's static PDFs input. The twobody near-field HIs part incorporated in the PA approximation lowers, additionally to the RP far-field part, the value of $D^{L}$. This additional slowing originates mainly from the shorttime self-friction coefficient $\zeta^{S}$ which in PA approximation is larger than its infinite dilution value $\zeta^{0}$. Figure 5 displays also experimental data for the cationic and anionic long-time self-diffusion coefficients of an aqueous $\mathrm{NaCl}$ solution. ${ }^{46}$ The concentration dependence of $D^{L}$ predicted theoretically on the basis of the RP treatment of the HIs nicely interpolates, for the employed mean ion diameter $\sigma=4.58 \AA$, between the measured diffusion coefficients of the smaller $\mathrm{Na}^{+}$cations (where $\sigma_{\mathrm{Na}^{+}}=3.62 \AA$ ) and the larger $\mathrm{Cl}^{-}$anions (where $\sigma_{\mathrm{Cl}^{-}}$ $=5.54 \AA$ ). This illustrates our general observation that the experimental conduction-diffusion and viscosity data for $\mathrm{NaCl}$ are consistently well described if HIs are treated on the RP level where, different from the more elaborate PA approxima- tion, the positive definiteness of the hydrodynamic diffusivity tensors is guaranteed.

While equal-sized cations and anions have been assumed for simplicity, we point out that the MCT-HIs can be generalized to differently sized ions. The resulting expressions for the transport properties are then more complicated, owing to the appearance of cross terms which are absent if all ions are of same size. A theoretical study of size-asymmetric electrolyte solutions requires in addition a more elaborate static input for the PDFs than the Attard or MSA solutions. Such a study will be described in a future communication.

\section{Cross correlation mobility part}

So far, we have dealt with the short-time contributions to the steady-state mobility matrix, $\boldsymbol{\mu}^{L}$, of an $m$-component electrolyte mixture of equal-sized ions described by MSA or Attard PDFs, and with the self-diffusion mobility part. We investigate in the following the general form of the relaxation part, $\Delta \boldsymbol{\mu}^{L}$, of the mobility matrix which according to Eq. (32) is related to the time-integrated, zero- $q$ limit of the collective memory function matrix. An additional discussion of the self- and cross-correlation contributions to $\Delta \boldsymbol{\mu}^{L}$ is given in Appendix B.

In the simplified MCT-HIs approach for the memory function matrix, we obtain the result

$$
\frac{1}{D^{S}} m_{\alpha \beta}^{c, i r r}=\left(\frac{\Delta \zeta_{H S}}{\zeta^{S}}+z_{\alpha} z_{\beta} \frac{\Delta \zeta_{E L}}{\zeta^{S}}\right) Q_{\alpha \beta}+M^{(m i x)} P_{\alpha \beta},
$$

where $Q_{\alpha \beta}=\delta_{\alpha \beta}-P_{n, \alpha \beta}-P_{\alpha \beta}$ are the elements of the projector matrix defined in Eq. (12), and

$$
\begin{aligned}
M^{(m i x)}= & \frac{\phi_{T}}{\pi^{3}} \int_{0}^{\infty} d y y^{2} \\
& \times \frac{\left[S_{E L}(y) h_{H S}(y)-\overline{z^{2}} S_{H S}(y) h_{E L}(y)\right]^{2}}{S_{E L}(y)\left[H_{N N}(y) / D^{S}\right]+S_{H S}(y)\left[H_{Z Z}(y) /\left(\overline{z^{2}} D^{S}\right)\right]} .
\end{aligned}
$$

The superscript (mix) is a reminder that there are mixed electro-steric and hard-sphere contributions in the integrand of Eq. (55). The self-correlation part of the diagonal matrix elements, $m_{\alpha \alpha}^{c, i r r}$, given by

$$
\frac{1}{D^{S}}\left(m_{\alpha \alpha}^{\mathrm{c}, \text { irr }}\right)^{\text {self }}=\frac{\Delta \zeta_{\alpha}}{\zeta^{S}}=\frac{D^{S}}{D_{\alpha}^{L}}-1,
$$

is related to self-correlations in the velocity of an $\alpha$-type ion. As we have noted in relation to Eq. (49), the self-correlation part follows from singling out a small fraction of $\alpha$-type ions considered to form a separate component $\alpha^{*}$ with $n_{\alpha^{*}} \rightarrow 0$. Velocity cross correlations between ions of the same or different components are related to the cross-correlation memory matrix part with elements

$$
\begin{aligned}
\frac{1}{D^{S}}\left(m_{\alpha \beta}^{\mathrm{c}, \text { irr }}\right)^{\text {cross }}= & M^{(m i x)} P_{\alpha \beta}-\left(\frac{\Delta \zeta_{H S}}{\zeta^{S}}+z_{\alpha} z_{\beta} \frac{\Delta \zeta_{E L}}{\zeta^{S}}\right) \\
& \times\left(P_{n, \alpha \beta}+P_{\alpha \beta}\right) .
\end{aligned}
$$


In the point-ion limit, it is $\Delta \zeta_{H S}=0$ and $M^{(m i x)}=\overline{z^{2}} \Delta \zeta_{E L}$. The time-integrated memory function matrix reduces then to the limiting law expression

$$
\lim _{\sigma \rightarrow 0} \frac{1}{D^{S}} m_{\alpha \beta}^{\mathrm{c}, \text { irr }}=\frac{\Delta \zeta_{E L}}{\zeta^{0}}\left(\delta_{\alpha \beta}-P_{\alpha \beta}\right) z_{\alpha} z_{\beta},
$$

where the ratio $\Delta \zeta_{E L} / \zeta^{0}$ is given for point ions in Eq. (I.135).

To obtain $\Delta \boldsymbol{\mu}$ using Eq. (32), the inverse of the matrix $\mathbf{D}=\mathbf{1}+\mathbf{H}^{-1} \cdot \mathbf{m}^{c, \text { irr }}$ is required. An algebraic procedure to obtain this inverse which in addition allows for systematically separating self- from cross-correlation contributions, is sketched in Appendix B. This procedure is useful also in dealing with electrolytes consisting of more than two ionic species.

In the remainder of this section, we present semi-analytic results for conduction-diffusion properties of binary electrolytes composed of equal-size spheres.

\section{Electrophoretic mobilities of a binary electrolyte}

While the analytic determination of the short-time part, $\boldsymbol{\mu}^{S}=\mathbf{H} / k_{B} T$, of the mobility matrix $\boldsymbol{\mu}^{L}$ is rather straightforward even for a general $m$-component electrolyte solution, the analysis of the relaxation part, $\Delta \boldsymbol{\mu}$, is more cumbersome, as it is noticed from Eq. (B9).

To make further analytic progress, in the remainder of this section we restrict ourselves to a binary $z_{c}: z_{a}$ electrolyte ( $c$ stands for cation, and $a$ for anion) of equal-sized ions which can be charge asymmetric in general. Total electroneutrality implies for a binary electrolyte that $P_{n, \alpha \beta}+P_{\alpha \beta}=\delta_{\alpha \beta}$, so that $Q_{\alpha \beta}=0$ for $\alpha, \beta \in\{a, c\}$. Thus, the time-integrated $2 \times 2$ memory function matrix of a binary electrolyte with MSA or Attard PDFs is given by

$$
\mathbf{m}^{c, i r r}=D^{S} M^{(m i x)} \mathbf{P},
$$

i.e., the time-integrated memory function matrix is proportional to the $2 \times 2$ projector, $\mathbf{P}$, on the subspace of chargedensity fluctuations. Using this result, $\boldsymbol{\mu}^{L}$ is obtained as

$$
\frac{k_{B} T}{D^{0}} \boldsymbol{\mu}^{L}=K_{H S}^{\text {sed }} \mathbf{P}_{n}+\frac{H_{Z Z}}{\overline{z^{2}} D^{0}} \frac{\frac{H_{Z Z}}{\bar{z}^{2} D^{S}}}{\frac{H_{Z Z}}{z^{2} D^{S}}+M^{(m i x)}} \mathbf{P} .
$$

In the (formal) limit of electrically neutral spheres, Eq. (60) reduces to

$$
k_{B} T \boldsymbol{\mu}^{L}=D^{0} K_{H S}^{\text {sed }} \mathbf{P}_{n} .
$$

This result is in accordance with the fact that the longtime sedimentation coefficient of a homogeneous system of monodisperse Brownian spheres, which is the long-time mean sedimentation velocity divided by its infinite dilution value, includes no slowing relaxation effect (i.e., memory matrix) contribution, provided the HIs can be treated on the pairwise additive level of approximation. ${ }^{33,40}$ Incidentally, the relative difference of the short-time and long-time sedimentation velocities for a monodisperse neutral hard-sphere system is quite small, amounting to less than 7\%, even for large particle volume fractions up to $\phi_{T} \lesssim 0.45$ where many-body HIs are in general strong. ${ }^{49,50}$ Further discussion on this subject is given in Appendix C.
In the point-ion limit, Eq. (60) reduces to

$$
\frac{k_{B} T}{D^{0}} \boldsymbol{\mu}^{L}=\mathbf{P}_{n}+\frac{\left(1-\frac{D_{\kappa}}{D^{0}}\right)^{2}}{1-\frac{D_{\kappa}}{D^{0}}+\overline{z^{2}} f^{e h}(\kappa) \kappa L_{B}} \mathbf{P},
$$

with the function $f^{e h}(\kappa)$ given in Eq. (I.121). Using $f^{e h}(0)$ $=f^{p}$, and on realizing that for a binary electrolyte $P_{c c}$ $=z_{c} /\left(z_{c}-z_{a}\right)=x_{a}, P_{a a}=1-P_{c c}$, and $P_{a c}=-\left(x_{a} x_{c}\right)^{1 / 2}$, the DFOF limiting law results for the mobility matrix constituents in Eqs. (I.86), (I.98), and (I.99) are recovered.

The steady-state electrophoretic mobility of a binary electrolyte follows straightforwardly from Eq. (60) as

$$
\mu_{\alpha}^{e l}=\frac{H_{Z Z} /\left(\overline{z^{2}} D^{S}\right)}{H_{Z Z} /\left(\overline{z^{2}} D^{S}\right)+M^{(m i x)}} \mu_{\alpha}^{e l, S}
$$

with $\alpha \in\{a, c\}$. The cross-correlation part of the electrophoretic mobility is

$$
\left(\mu_{\alpha}^{e l}\right)^{\text {cross }}=\frac{H_{Z Z} /\left(\overline{z^{2}} D^{S}\right)}{H_{Z Z} /\left(\overline{z^{2}} D^{S}\right)+M^{(m i x)}} \mu_{\alpha}^{e l, S}-\frac{z_{\alpha} e}{k_{B} T} D_{\alpha}^{L},
$$

where $\mu_{\alpha}^{e l, S}$ is the short-time electrophoretic mobility given in Eq. (40).

Note that according to Eq. (I.41),

$$
\mu_{\alpha}^{e l}=\mu_{\alpha}^{e l, 0}+\Delta \mu_{\alpha}^{e l, S}+\Delta \mu_{\alpha}^{e l},
$$

the steady-state electrophoretic mobility is the sum of a single-ion part, $\mu_{\alpha}^{e l, 0}$, a short-time part of purely hydrodynamic origin,

$$
\frac{\Delta \mu_{\alpha}^{e l, S}}{\mu_{\alpha}^{e l, 0}}=-\left(1-\frac{H_{Z Z}}{\overline{z^{2}} D^{0}}\right),
$$

commonly referred to as the electrophoretic mobility part, and a relaxation part given by

$$
\frac{\Delta \mu_{\alpha}^{e l}}{\mu_{\alpha}^{e l, 0}}=-\frac{M^{(m i x)}}{M^{(m i x)}+H_{Z Z} /\left(\overline{z^{2}} D^{S}\right)} \frac{H_{Z Z}}{\overline{z^{2}} D^{0}} .
$$

Both the electrophoretic and relaxation parts reduce the magnitude of the electrophoretic ion mobility below its infinite dilution value. The relative strength of relaxation and short-time electrophoretic mobility contributions is quantified for a binary electrolyte by the ratio

$$
\begin{aligned}
& \frac{\Delta \mu_{\alpha}^{e l}}{\Delta \mu_{\alpha}^{e l, S}} \\
& =\frac{\Delta \mu_{\alpha}^{e l, S}+\Delta \mu_{\alpha}^{e l}}{\Delta \mu_{\alpha}^{e l, S}}-1 \\
& =\left(1-\frac{H_{Z Z}}{\overline{z^{2}} D^{0}} \frac{H_{Z Z} /\left(\overline{z^{2}} D^{S}\right)}{M^{(m i x)}+H_{Z Z} /\left(\overline{z^{2}} D^{S}\right)}\right) /\left(1-\frac{H_{Z Z}}{\overline{z^{2}} D^{0}}\right)-1,
\end{aligned}
$$

which is independent of the component index $\alpha \in\{a, c\}$.

The mobility ratio for a symmetric 1:1 electrolyte is plotted in Fig. 6. Results based on RP-HIs and PA-HIs, respectively, are compared to each other. The relaxation part, $\Delta \mu_{\alpha}^{e l}$, has been calculated using the simplified MCT-HIs method. Its relative importance depends on the employed treatment 


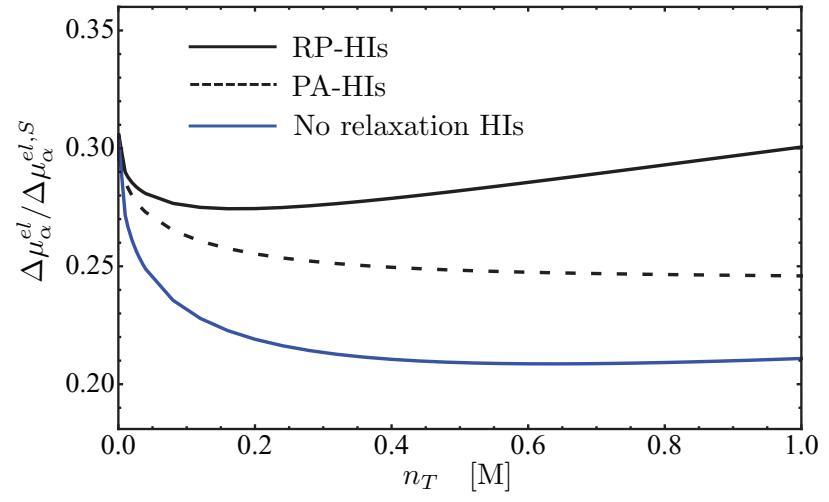

FIG. 6. Ratio of the relaxation electrophoretic mobility part, $\Delta \mu_{\alpha}^{e l}$, determined by the MCT-HIs method, and the short-time electrophoretic mobility part, $\Delta \mu_{\alpha}^{e l, S}$, of a 1:1 electrolyte with parameters as in Fig. 2, and using the Attard static input. By symmetry, the ratio is the same for cations and anions. Solid black line: Result obtained using the RP-HIs approximation both in $\Delta \mu_{\alpha}^{e l, S}$ and $\Delta \mu_{\alpha}^{e l}$. Dashed black: Result for PA-HIs used both for $\Delta \mu_{\alpha}^{e l, S}$ and $\Delta \mu_{\alpha}^{e l}$. Blue solid line: Mobility ratio for RP-HIs used in $\Delta \mu_{\alpha}^{e l, S}$, and HIs disregarded in $\Delta \mu_{\alpha}^{e l}$.

of the HIs. The ratio is largest when the HIs are treated on the RP level, for which in the depicted concentration range $\Delta \mu_{\alpha}^{e l}$ amounts, on average, to about one third of the shorttime electrophoretic mobility part. The comparison with the MCT-predicted ratio where HIs are neglected in the relaxation part but maintained on the RP level for $\Delta \mu_{\alpha}^{e l, S}$ (bottom blue curve) shows that HIs significantly enlarge the relaxation mobility part. The mobility ratio based on RP-HIs has a minimum at $n_{T} \approx 0.1 \mathrm{M}$, corresponding to $\kappa \sigma \approx 0.34$. With increasing concentrations of values larger than the concentration at the minimum, the mobility ratio increases slowly. In contrast, the mobility ratio based on PA-HIs employed in the relaxation and short-time parts decreases monotonically with increasing concentration. The significantly smaller mobility ratio for PA-HIs is basically a consequence of the underestimation of $D^{L}$ in the PA approximation (see Fig. 5). For $n_{T}$ $\rightarrow 0$, the mobility ratio converges to $f^{p} L_{B} / a \approx 0.3$. This limiting value is independent of the HIs.

In the point-ion limit, Eq. (63) reduces to

$$
\frac{\mu_{\alpha}^{e l}}{\mu_{\alpha}^{e l, 0}}=\frac{\left(1-\frac{D_{\kappa}}{D^{0}}\right)^{2}}{\left(1-\frac{D_{\kappa}}{D^{0}}\right)+\overline{z^{2}} f^{e h}(\kappa) \kappa L_{B}},
$$

which if expanded to first order in $\kappa$ simplifies further to the DFOF limiting law result, ${ }^{5}$

$$
\mu_{\alpha}^{e l}=\mu_{\alpha}^{e l, 0}-\frac{z_{\alpha} e \kappa}{6 \pi \eta_{0}}-\overline{z^{2}} f^{p} \kappa L_{B} \mu_{\alpha}^{e l, 0} .
$$

The low-concentration relaxation effect contribution to the electrophoretic mobility in Eq. (70) proportional to $f^{p}$ is determined by Coulomb forces alone, independent of the HIs.

The DFOF limiting law self-correlation and crosscorrelation contributions to the total electrophoretic mobility written in the form

$$
\mu_{\alpha}^{e l}=\left(\mu_{\alpha}^{e l}\right)^{\text {self }}+\left(\mu_{\alpha}^{e l}\right)^{\text {cross }}
$$

are deduced from Eq. (70) by noting that $\overline{z^{2}}=-z_{c} z_{a}$ and $-z_{\alpha}^{2}+\overline{z^{3}} z_{\alpha} \overline{z^{2}}=-\overline{z^{2}}$ for $\alpha=\{c, a\}$. This leads to the DFOF expressions in Eqs. (I.102) and (I.103) for the self-correlation and cross-correlation parts, respectively, in Eq. (71).

\section{E. Molar conductivity of a binary electrolyte}

The molar conductivity, $\Lambda$, follows immediately from its definition for strong electrolytes given by Eq. (I.8), and here rewritten

$$
\Lambda=\sum_{\alpha=1}^{m} v_{\alpha} \lambda_{\alpha}^{e l}=\mathcal{F} \sum_{\alpha=1}^{m} v_{\alpha} z_{\alpha} \mu_{\alpha}^{e l}
$$

where

$$
\lambda_{\alpha}^{e l}=z_{\alpha} \mu_{\alpha}^{e l} \mathcal{F}
$$

is the partial molar conductivity proportional to the stoichiometric coefficient, $v_{\alpha}$, of $\alpha$-type ions. Here, $\mathcal{F}=N_{A} e$ is Faraday's constant and $N_{A}$ is Avogadro's number.

According to Eqs. (40) and (63), the dependence of the steady-state electrophoretic mobility $\mu_{\alpha}^{e l}$ on the component index $\alpha$ is due to the factor $\mu_{\alpha}^{e l, 0}$ only. Therefore, we obtain

$$
\lambda_{\alpha}^{e l}=\left(\frac{H_{Z Z} /\left(\overline{z^{2}} D^{S}\right)}{H_{Z Z} /\left(\overline{z^{2}} D^{S}\right)+M^{(m i x)}}\right) \frac{H_{Z Z}}{D^{0} \overline{z^{2}}} \lambda_{\alpha}^{e l, 0},
$$

for the steady-state partial molar conductivity, leading to

$$
\frac{\Lambda}{\Lambda^{0}}=\left(\frac{H_{Z Z} /\left(\overline{z^{2}} D^{S}\right)}{H_{Z Z} /\left(\overline{z^{2}} D^{S}\right)+M^{(m i x)}}\right) \frac{\Lambda^{S}}{\Lambda^{0}},
$$

for the molar conductivity of a binary electrolyte solution normalized by its infinite dilution value $\Lambda^{0}$. The latter is given by Eq. (72) with $\lambda_{\alpha}^{e l}$ replaced by $\lambda_{\alpha}^{e l, 0}$, and $\mu_{\alpha}^{e l}$ by $\mu_{\alpha}^{e l, 0}$.

The short-time partial molar conductivity, $\lambda_{\alpha}^{e l, S}$, is given by Eq. (74), but with vanishing memory function part $M^{(m i x)}$. This implies

$$
\frac{\Lambda^{S}}{\Lambda^{0}}=\frac{H_{Z Z}}{\overline{z^{2}} D^{0}}
$$

for the normalized short-time molar conductivity $\Lambda^{S} / \Lambda^{0}$ which, according to Eq. (40), is equal to the normalized shorttime electrophoretic mobility $\mu_{\alpha}^{e l, S} / \mu_{\alpha}^{e l, 0}$. It is evident from Eq. (75) that $\Lambda<\Lambda^{S}$ for $n_{T}>0$.

The concentration dependence of $\Lambda^{S} / \Lambda^{0}$ for a symmetric 1:1 electrolyte corresponds to the blue solid and blued dashed lines depicted in Fig. 3. In accordance with a Brownian dynamics simulation study, ${ }^{51}$ the conductivity is significantly decreased if HIs are taken into account. Note here that $\Lambda^{S}$ $=\Lambda^{0}$ if HIs are disregarded.

In the point-ion limit, Eq. (75) reduces to

$$
\frac{\Lambda}{\Lambda^{0}}=\frac{\left(1-\frac{D_{\kappa}}{D^{0}}\right)^{2}}{\left(1-\frac{D_{\kappa}}{D^{0}}\right)+\overline{z^{2}} f^{e h}(\kappa) \kappa L_{B}} .
$$

By expanding Eq. (77) to first order in $\kappa$, the DFOF limiting law result for the molar conductivity, ${ }^{5}$

$$
\Lambda=\Lambda^{0}-\overline{z^{2}} f^{p} \kappa L_{B} \Lambda^{0}-\left(\sum_{\alpha=1}^{m} v_{\alpha} z_{\alpha}^{2}\right) \frac{e \mathcal{F} \kappa}{6 \pi \eta_{0}},
$$

is recovered. 
When the electrophoretic mobility in Eq. (72) is approximated by its self-correlation part (cf. Eq. (36)), the wellknown Nernst-Einstein (NE) relation,

$$
\Lambda \approx \frac{\mathcal{F}^{2}}{R T} \sum_{\alpha=1}^{m} v_{\alpha} z_{\alpha}^{2} D_{\alpha}^{L},
$$

follows, where $R=N_{A} k_{B}$. For a symmetric binary electrolyte, the NE relation has the compact form,

$$
\frac{\Lambda}{\Lambda^{0}} \approx \frac{D^{L}}{D^{0}},
$$

i.e., the molar conductivity in this approximate relation is taken to be proportional to the long-time self-diffusion coefficient, $D^{L}$, of cations and anions. In assuming the validity of the NE relation between the collective property $\Lambda$ and the self-dynamics property $D^{L}$, dynamic cross-correlations in the ion velocities are neglected which make the actual value of $\Lambda / \Lambda^{0}$ smaller than that of $D^{L} / D^{0}$. From comparing the DFOF expression for $D^{L}$ in Eq. (53) with the DFOF electrophoretic mobility in Eq. (70), one notices that the NE relation is approximate already to first order in $\kappa$.

In Fig. 7, we compare our simplified MCT-HIs predictions for the normalized conductivity of an aqueous 1:1 electrolyte with that by Chandra et al. ${ }^{26}$ as obtained from their MCT-DDFT approach with the ion diameters of cations and anions taken equal to the Pauling radius values of sodium and chloride, respectively (green solid line). The MCT-HIs conductivity curves with RP-HIs (black solid line) and PAHIs (black dashed line), respectively, are only moderately different from each other for the small mean ion diameter employed here. Neglecting the RP-HIs in the relaxation mobility contribution, while keeping them in the short-time (electrophoretic) part, gives rise to a moderate increase in the molar

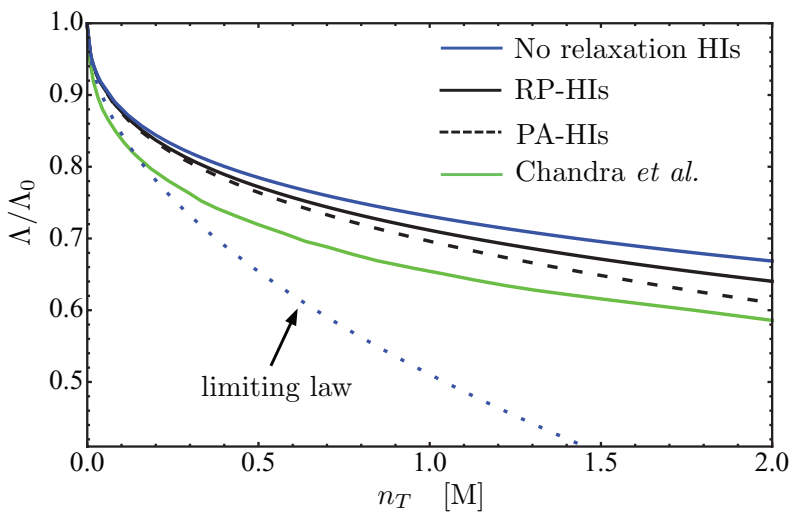

FIG. 7. Concentration dependence of the normalized molar conductivity, $\Lambda / \Lambda^{0}$, of an aqueous 1:1 electrolyte solution at room temperature. For the here depicted simplified MCT-HIs results, equal-sized cations and anions of diameter $\sigma=2.82 \AA$ have been assumed. This value is very close to the sum of the Pauling ionic radii of $\mathrm{Na}^{+}(1.96 \AA)$ and $\mathrm{Cl}^{-}(3.62 \AA)$ used by Chandra et al. in their two-species MCT-DDFT conductivity prediction (green curve, reproduced from Fig. 1(b) in Chandra et al. ${ }^{26}$ ). Black solid curve: Simplified MCT-HIs result using RP-HIs in the relaxation and short-time parts. Black dashed curve: Simplified MCT-HIs result using PA-HIs in the relaxation and short-time parts. Blue solid line: MCT-HIs result using RP-HIs for in the short-time conductivity part $\Lambda^{S}$ only. Dotted blue line: DFOF limiting law result according to Eq. (78), specialized to monovalent ions. For all theoretical results shown in the figure, $D^{0}$ is determined from $D^{0}=k_{B} T /\left(3 \pi \eta_{0} \sigma\right)$.
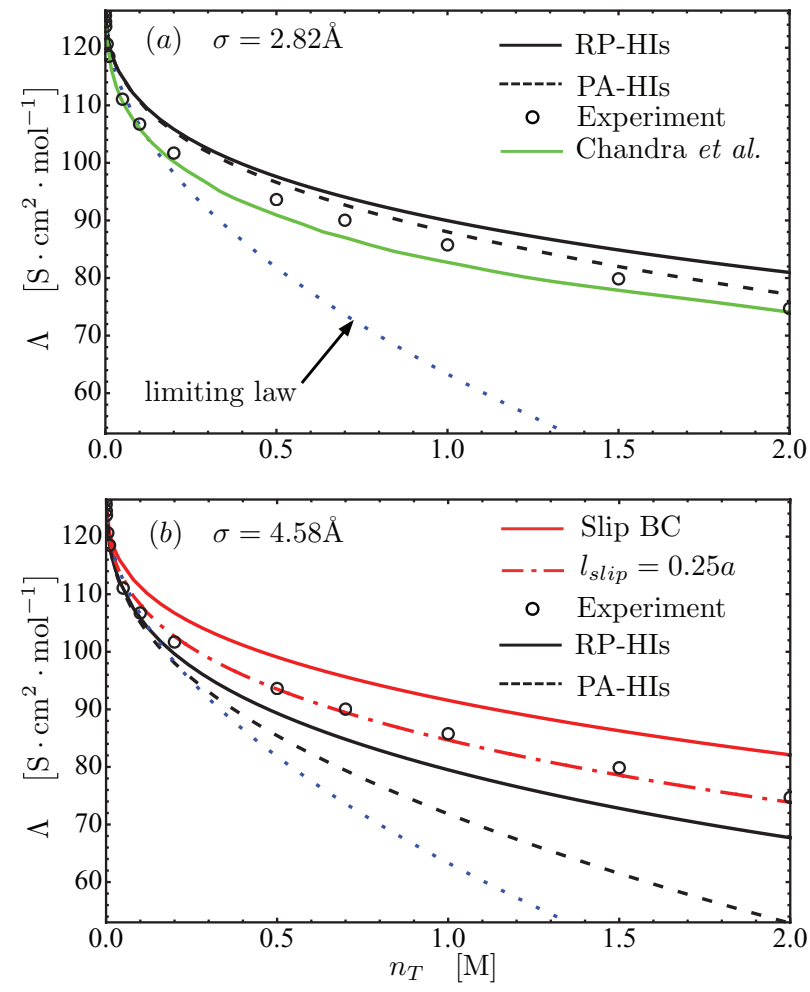

FIG. 8. Comparison of theoretical conductivity predictions with experimental values for the molar conductivity of $\mathrm{NaCl}$ in water at $T=25^{\circ} \mathrm{C} .{ }^{52}$ (a) Simplified MCT-HIs prediction using $\sigma=2.82 \AA$. Black line: MCT-HIs prediction for RP-HIs used both in $\Delta \mu_{\alpha}^{e l, S}$ and $\Delta \mu_{\alpha}^{e l}$. Dashed black line: MCTHIs prediction for PA-HIs used both in $\Delta \mu_{\alpha}^{e l, S}$ and $\Delta \mu_{\alpha}^{e l}$. Green line: MCTDDFT result by Chandra et al. ${ }^{26}$ where HIs are disregarded in the relaxation conductivity part. Dotted blue line: DFOF limiting law result according to Eq. (78). (b) MCT-HIs molar conductivity predictions using now the hydrodynamic mean ion diameter $\sigma=4.58 \AA$, consistent with the underlying PM model of hydrated electrolyte ions. Line code for the solid-black, dashedblack, and dotted-blue curves as in (a). The effect of mixed slip-stick BCs in the dipolar HIs treatment on the molar conductivity is illustrated for the perfect slip BC $(\xi=1 / 3$, solid red line), and for the slip parameter $\xi=1 / 7$ corresponding to the slip length $l_{p}=a / 4$ (red dashed-dotted line).

conductivity (blue solid curve). While similar in the concentration dependence, the MCT-HIs conductivity curves are located above the MCT-DDFT conductivity curve obtained by Chandra et al. ${ }^{26}$ In their MCT-DDFT approach, the influence of HIs on the ion atmosphere relaxation contribution to the conductivity has been disregarded.

In Fig. 8(a), the theoretical predictions for the molar conductivity are compared, on an absolute scale, with experimental data for an aqueous $\mathrm{NaCl}$ solution. ${ }^{52}$ The theoretical curves for $\Lambda$ have been obtained from multiplying the calculated conductivity ratio, $\Lambda / \Lambda^{0}$, with the experimental values for the molar conductivity of an ultra-dilute sodium-chloride solution. ${ }^{52}$ The simplified MCT-HIs curves for $\Lambda$ are located somewhat above the experimental data points, whereas the MCT-DDFT curve by Chandra et al. ${ }^{26}$ slightly underestimates the experimental data. We stress, however, that the diameter $\sigma=2.82 \AA$ is employed for the MCT-HIs results in Fig. 8(a) solely for the comparison with the conductivity result by Chandra et al. ${ }^{26}$ where the Pauling ion diameters have been used. In fact, to be consistent with the PM underlying the MCT-HIs scheme, the mean diameter $\sigma=4.58 \AA$ based 
on the sizes of the hydrated $\mathrm{Na}^{+}$and $\mathrm{Cl}^{+}$ions should be used instead. ${ }^{53}$ This latter diameter value has been employed in Figs. 2-6.

The comparison of the MCT-HIs results with the experimental molar conductivity data for $\mathrm{NaCl}$ in water, using $\sigma=4.58 \AA$ for the mean ion diameter, is given in Fig. 8(b). The MCT-HIs curve for RP-HIs is in good overall agreement with the experimental data, although the experimental values are underestimated to some extent for larger concentrations. The experimental data are strongly underestimated, however, if the HIs are treated on the PA-HIs level. Therefore, the RP approximation should be used in the simplified MCTHIs approach. While the MCT-DDFT conductivity curve for $\sigma=4.58 \AA$ is not given in the literature, this curve is expected to strongly underestimate the experimental conductivity data, owing to the fact that the total ion volume fraction in Fig. 8(b) is more than four times larger than that in Fig. 8(a).

The comparison of the MCT-HIs molar conductivity curve in Fig. 8(b) (black solid line) with the corresponding MCT-HIs result for $D^{L} / D^{0}$ in Fig. 5 illustrates that the usage of the NE relation in Eq. (80) can result in a strong overestimation of the molar conductivity. As pointed out earlier, this overestimation is due to the neglected dynamic cross correlations.

\section{EFFECTS OF HYDRODYNAMIC BOUNDARY CONDITIONS}

For the hydrodynamic diffusivity tensors used so far, the stick hydrodynamic BC on the PM ion surfaces has been assumed. The MCT-HIs method is straightforwardly generalized to Navier mixed slip-stick BCs, corresponding to weaker HIs. In the following, we investigate the implications for the molar conductivity and electrolyte viscosity when mixed slipstick BCs are used. These BCs allow for mimicking approximately, on a continuum level of description, the hydrodynamic influence of ion hydration shells. Explicit results for conduction-diffusion transport properties, and the electrolyte viscosity, are discussed in particular for the two extremal cases of stick and perfect slip BCs. In the latter case, the tangential hydrodynamic shear stress on the ion surfaces is taken to be zero. Regarding the sedimentation coefficient, in Appendix $\mathrm{C}$ we analyze, in addition, the implications of a possible solvent permeability of the hydrated ions. The HIs are treated in the following on the dipolar far-field level, i.e., the $\mathrm{RP}$ approximation with stick $\mathrm{BC}$ on the ion spheres is generalized to Navier mixed slip-stick hydrodynamic BCs (in Subsections IV A and IV B). In Appendix C, the sedimentation coefficient of an electrolyte solution of solvent-permeable, equal-sized ions is calculated. Therein the ions are described as uniformly permeable spheres characterized by a constant Darcy solvent permeability.

\section{A. Far-field Hls up to dipolar order}

We consider again a mixture of PM ions of identical hydrodynamic radius $a=\sigma / 2$ using now, however, general mixed slip-stick hydrodynamic BCs.
The translational diffusion coefficient, $D^{0}(\xi)$, of an isolated sphere with mixed slip-stick surface BCs characterized by the slip parameter $\xi \in\{0,1 / 3\}$, is given by ${ }^{54}$

$$
D^{0}(\xi)=\frac{k_{B} T}{6 \pi \eta_{0} a P_{1}^{(m)}(\xi)},
$$

where

$$
P_{1}^{(m)}(\xi)=1-\xi
$$

For $\xi=0$, the stick BC case is recovered for which $D^{0}(\xi$ $=0)=D^{0}$. The perfect slip BC corresponds to $\xi=1 / 3$ where $P_{1}(1 / 3)=2 / 3$ and $D^{0}(\xi=1 / 3)=3 D^{0} / 2$. No tangential hydrodynamic stress, and thus no hydrodynamic torque is exerted by the surrounding fluid on a perfect slip sphere. For values of $\xi$ in between 0 and $1 / 3$, there is a non-zero tangential slip velocity of the fluid relative to particle surface points, which is proportional to the local tangential hydrodynamic stress. The slip length, $l_{\text {slip }}$, associated with the slip parameter is given by

$$
\frac{l_{\text {slip }}}{a}=\frac{\xi}{1-3 \xi} .
$$

It is zero (infinite) for stick (perfect slip) BC (see Fig. 1).

The far-field form of the distinct diffusivity tensor, $\mathbf{D}_{12}^{\alpha \beta}$, of equal-sized ions $i$ and $j$ with mixed slip-stick $\mathrm{BC}$ is to $\mathcal{O}\left(1 / x^{3}\right)$ in the reduced distance $x$ given by ${ }^{55,56}$

$$
\mathbf{D}_{12}^{\alpha \beta}\left(\mathbf{R}_{i}^{\alpha}-\mathbf{R}_{j 2}^{\beta} ; \xi\right)=D^{0}(\xi) \mathbf{T}^{(m)}\left(\mathbf{R}_{i}^{\alpha}-\mathbf{R}_{j}^{\beta} ; \xi\right),
$$

where

$$
\begin{aligned}
\mathbf{T}^{(m)}(\mathbf{r} ; \xi)= & \frac{3 a}{4 r} P_{1}^{(m)}(\xi)(\mathbf{1}+\widehat{\mathbf{r}} \mathbf{r}) \\
& +\frac{1}{2}\left(\frac{a}{r}\right)^{3} P_{2}^{(m)}(\xi)(\mathbf{1}-3 \widehat{\mathbf{r}} \widehat{\mathbf{r}})
\end{aligned}
$$

is basically the generalized Rotne-Prager type tensor for mixed slip-stick BCs, and

$$
P_{2}^{(m)}(\xi)=1-3 \xi .
$$

We note here that $\mathbf{D}_{11}^{\alpha \beta}=D^{0}(\xi) \mathbf{1}+\mathcal{O}\left(1 / x^{4}\right)$ which implies that $D^{S}(\xi)=D^{0}(\xi)$ holds sway for the short-time selfdiffusion coefficient calculated to dipolar order in the HIs.

In the point-particle limit, or if the perfect slip BC is assumed, $D^{0}(\xi) \mathbf{T}^{(m)}(\mathbf{r} ; \xi)$ reduces to $k_{B} T \mathbf{T}^{O s}(\mathbf{r})$ where $\mathbf{T}^{O s}(\mathbf{r})$ is the Oseen tensor describing the fluid velocity response due to a unit point force exerted at $\mathbf{r}=\mathbf{0}$ to an infinite and unbounded fluid. ${ }^{34}$ The dipolar contribution to $\mathbf{T}^{(m)}$ is zero for the perfect slip BC, so that in this case no curvature contribution arises in presence of nonlinear fluid flow. For the stick BC, the Rotne-Prager tensor, $\mathbf{T}^{R P}(\mathbf{r})$, of equal-sized spheres ${ }^{37}$ is recovered from Eq. (85). The explicit expression of this tensor is given in Eq. (I.74).

The hydrodynamic function matrix, $\mathbf{H}(y)$, for equal-sized ions with mixed slip-stick $\mathrm{BCs}$ is formally still given by Eqs. (A11)-(A13). However, to dipolar order in the HIs, the global hydrodynamic functions are now

$$
H_{N N}(y ; \xi) / D^{0}(\xi)=1+\phi_{T} \mathrm{~h}_{H S}^{d,(m)}(y ; \xi)
$$

and

$$
\frac{H_{Z Z}(y ; \xi)}{\overline{z^{2}} D^{0}(\xi)}=1+\phi_{T} \mathrm{~h}_{E L}^{d,(m)}(y ; \xi)
$$


with the distinct hydrodynamic function contributions

$$
\begin{aligned}
\mathrm{h}_{H S}^{d,(m)}(y ; \xi)= & 3\left[P_{2}(\xi)-6 P_{1}(\xi)\right] \frac{j_{1}(y)}{y} \\
& +18 \int_{1}^{\infty} d x \times K^{(m)}(y, x ; \xi) h_{H S}(x) \\
= & P_{2}(\xi)+18 \int_{0}^{\infty} d x x K^{(m)}(y, x ; \xi) h_{H S}(x)
\end{aligned}
$$

and

$$
\mathrm{h}_{E L}^{d,(m)}(y ; \xi)=18 \int_{0}^{\infty} d x x K^{(m)}(y, x ; \xi) h_{E L}(x) .
$$

The mixed slip-stick hydrodynamic kernel is here

$$
\begin{aligned}
K^{(m)}(y, x ; \xi)= & P_{1}(\xi)\left(j_{0}(y x)-\frac{j_{1}(y x)}{y x}\right) \\
& +\frac{P_{2}(\xi)}{6}\left(\frac{j_{2}(y x)}{x^{2}}\right) .
\end{aligned}
$$

In the zero slip case $\xi=0$, the RP-HIs forms of $H_{N N}$ and $H_{Z Z}$ are recovered from these expressions.

Next, consider uniformly permeable spheres of radius $a$ (with associated quantities labeled by the superscript $(p)$ ), where the interior average fluid flow is described by the Brinkman-Debye-Bueche equation. ${ }^{57}$ The parameter characterizing to what extent a sphere is solvent permeable is the ratio, $\chi$, of particle radius and hydrodynamic penetration depth. The latter is equal to the square-root of the Darcy permeability.

The diffusion coefficient, $D^{0}(\chi)$, of an isolated and uniformly permeable sphere follows from Eq. (81) by replacing $P_{1}^{(m)}(\xi)$ by the function

$$
P_{1}^{(p)}(\chi)=\frac{2 \chi^{2}[1-\tanh (\chi) / \chi]}{2 \chi^{2}+3[1-\tanh (\chi) / \chi]} .
$$

Likewise, the $\mathcal{O}\left(1 / x^{3}\right)$ dipolar form of the distinct diffusivity tensor of uniformly permeable spheres, $D^{0}(\chi) \mathbf{T}^{(p)}(\mathbf{r}, \chi)$, is given by Eqs. (84) and (85) with $P_{1}^{(m)}(\xi)$ replaced by $P_{1}^{(p)}(\chi)$, and $P_{2}^{(m)}(\xi)$ by ${ }^{57-59}$

$$
P_{2}^{(p)}(\chi)=\frac{6\left(\chi^{2}-2\right)[\chi-\tanh (\chi)]-4 \chi^{3}}{2 \chi^{3}+3[\chi-\tanh (\chi)]} .
$$

In the limit $\chi \rightarrow \infty$ of zero hydrodynamic penetration depth, it is $P_{1}^{(p)}(\infty)=1=P_{2}^{(p)}(\infty)$, and the hydrodynamic functions of impermeable spheres with stick BC are recovered.

For a system of equally sized uniformly permeable spheres, expressions for the global hydrodynamic functions $H_{N N}$ and $H_{Z Z}$ are obtained completely analogous to Eqs. (87)(91), simply from replacing $P_{i}^{(m)}(\xi)$ by $P_{i}^{(p)}(\chi)$ for $i=1,2$. Transport properties of a concentrated permeable hard-sphere system have been intensely studied in the past years, ${ }^{60,61}$ since this system serves as a minimal model for permeable colloidal particle systems such as core-shell particles consisting of a dry core and a permeable shell (e.g., a polymer brush). ${ }^{62,63}$ In typical core-shell colloidal systems, the inverse reduced permeability parameter is in the range ${ }^{62} \chi \gtrsim 20$. Appendix $C$ presents results for the sedimentation coefficient of a dispersion of monodisperse, solvent-permeable (ion) spheres, and of a dispersion of mixed stick-slip BC spheres.

\section{B. Viscosity and conductivity: Mixed slip-stick Navier BCs}

We discuss first the implications for the short-time molar conductivity on using mixed slip-stick BCs. In generalizing Eq. (76) to mixed slip-stick BCs, $\Lambda_{S}$ is given by

$$
\frac{\Lambda^{S}(\xi)}{\Lambda^{0}(\xi)}=\frac{H_{Z Z}(0 ; \xi)}{\overline{z^{2}} D^{0}(\xi)} \text {. }
$$

The normalized conductivity, $\Lambda^{S}(\xi) / \Lambda^{0}(\xi)$, is largest for the perfect slip BC where the tangential hydrodynamic surface shear stress is zero, and smallest for stick BC. From

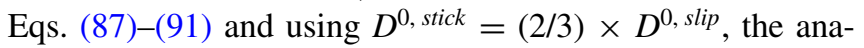
lytic expression

$$
1-\left(\frac{\Lambda^{S}}{\Lambda^{0}}\right)^{\text {slip }}=\frac{2}{3}\left[1-\left(\frac{\Lambda^{S}}{\Lambda^{0}}\right)^{\text {stick }}\right]
$$

is obtained relating the two limiting short-time conductivities. This relation makes it transparent that $\left(\Lambda^{S} / \Lambda^{0}\right)^{s l i p}$ $>\left(\Lambda^{S} / \Lambda^{0}\right)^{\text {stick }}$.

A corresponding analytic relation between the steadystate molar conductivities for stick and perfect slip BCs is not known. The steady-state conductivity is smaller than its short-time part, owing to the slowing effect of the ion cloud relaxation. This exemplifies a general rule valid in Smoluchowski dynamics which tells that a steady-state (long-time) linear transport coefficient is smaller than its short-time part, since the former is related to the time integral of an associated time autocorrelation function decaying strictly monotonically in Smoluchowski dynamics (see Paper $\mathrm{I}^{85}$ ). The steady-state and short-time coefficients are equal in the infinite dilution limit only.

The MCT-HIs predictions for the molar conductivity with mixed-slip BCs, and HIs treated on the dipolar far-field level, are included in Fig. 7(b). While the conductivity prediction for stick BC is in decently good agreement with the experimental data of $\mathrm{NaCl}$ in water in particular at lower concentrations, a very good fit of the experimental data is achieved using the slip parameter $\xi=1 / 7$. This value corresponds, according to Eq. (83), to the slip length $l_{\text {slip }}=a / 4$ comparable to the average thickness of the ion hydration layers.

We discuss next the effect on the electrolyte viscosity of using mixed slip-stick BCs. The viscosity is the sum,

$$
\eta=\eta_{0}+\Delta \eta_{\text {exc }}
$$

of the solvent viscosity $\eta_{0}$ and the excess part $\Delta \eta_{\text {exc }}$. The latter is determined by the ion-ion direct and hydrodynamic interactions. The excess viscosity part in turn consists of the high-frequency, i.e., short-time, contribution, $\Delta \eta_{\infty}$, and the contribution $\Delta \eta$ arising from the relaxation of the ion clouds under weak solution shear flow, i.e.,

$$
\Delta \eta_{e x c}=\Delta \eta_{\infty}+\Delta \eta .
$$


The high-frequency contribution, $\Delta \eta_{\infty}$, is of purely hydrodynamic origin. It vanishes for point particles or when HIs are disregarded. ${ }^{30}$

In previous work, ${ }^{30}$ we have derived a simplified MCTHIs expression for the steady-state electrolyte viscosity, $\eta$, of a mixture of equal-sized ions. Similar to the corresponding behavior of the short-time ionic self-diffusion coefficient, for equal-sized ions with pairwise additive HIs and the static PDFs treated on the MSA or Attard level, $\Delta \eta_{\infty}$ is equal to the high-frequency viscosity contribution, $\Delta \eta_{\infty}^{H S}$, of a neutral hard-sphere dispersion at volume fraction $\phi_{T}$. This equality is not valid for solutions of differently sized ions. For hard spheres with stick hydrodynamic $\mathrm{BC}$ is

$$
\begin{aligned}
\frac{\Delta \eta_{\infty}^{H S, \text { stick }}}{\eta_{0}} & =\frac{5}{2} \phi_{T}\left(1+\phi_{T}\right)+60 \phi_{T}^{2} \int_{1}^{\infty} d x x^{2} g_{H S}(x) J(x) \\
& =\frac{5}{2} \phi_{T}+5.0023 \phi_{T}^{2}+\mathcal{O}\left(\phi_{T}^{3}\right) .
\end{aligned}
$$

Here, $J(x)$ is the two-body hydrodynamic shear mobility function for stick BC, decaying like $\mathcal{O}\left(1 / x^{6}\right)$ at large $x$, and $g_{H S}(x)$ is the hard-sphere PDF. The second numerical equality in Eq. (98) has been obtained using a numerical table for $J(x){ }^{36}$ It agrees with an earlier second-order virial numerical result by Cichocki et al. ${ }^{64}$ where a different method was used. For the ion concentrations considered in the present work where $\phi_{T} \lesssim 0.06$ (see upper horizontal scale in Fig. 3), Eq. (98) is a good description ${ }^{40}$ for $\Delta \eta_{\infty}^{H S \text {,stick }}$.

The corresponding second-order virial expansion expression for the high-frequency viscosity contribution of hard spheres with mixed slip-stick BCs is ${ }^{65}$

$$
\frac{\Delta \eta_{\infty}^{H S,(m)}(\xi)}{\eta_{0}}=[\eta]^{(m)} \phi_{T}+k_{H}^{(m)}\left([\eta]^{(m)}\right)^{2} \phi_{T}^{2}+\mathcal{O}\left(\phi_{T}^{3}\right),
$$

where

$$
[\eta]^{(m)}=\frac{5}{2} \cdot \frac{1-\xi}{1+2 \xi}
$$

is the single-sphere intrinsic viscosity, and $k_{H}^{(m)}$ is the twoparticle Huggins coefficient for mixed slip-stick BCs spheres. The Huggins coefficient has been numerically determined to high accuracy as a function of $\xi$ by Cichocki and Felderhof. ${ }^{65}$ Their result is well parameterized by the fifth-order polynomial

$$
\begin{aligned}
k_{H}^{(m)}\left([\eta]^{(m)}\right)^{2}= & 5-12.8694 s+21.4608 s^{2} \\
& -25.4771 s^{3}+17.4982 s^{4}-4.90946 s^{5},
\end{aligned}
$$

in $s=3 \xi$, which we have obtained from a least-square fit of the numerical data by Cichocki and Felderhof. ${ }^{65}$ For stick BC where $[\eta]^{(m)}(\xi=0)=5 / 2$, Eq. (99) reduces to Eq. (98). For perfect slip BC is $[\eta]^{(m)}(\xi=1 / 3)=1$, and the second-order virial result for hydrodynamically perfectly slip spheres, ${ }^{65}$

$$
\frac{\Delta \eta_{\infty}^{H S, \text { slip }}}{\eta_{0}}=\phi_{T}+0.703 \phi_{T}^{2}+\mathcal{O}\left(\phi_{T}^{3}\right)
$$

is recovered from Eq. (99). Even for perfect slip spheres, the intrinsic viscosity value $\left([\eta]^{s l i p}=1\right)$ is non-zero, owing to the fact that fluid cannot flow through an impermeable particle.
For equal-sized ions described using the Attard or MSA PDFs, the shear relaxation viscosity contribution in the simplified MCT-HIs approximation is given by the sum, ${ }^{30}$

$$
\Delta \eta=\Delta \eta_{H S}+\Delta \eta_{E L},
$$

of a pure hard-sphere and an electrosteric part. For mixed slipstick BCs, the simplified MCT-HIs expressions for the two parts are

$$
\frac{\Delta \eta_{H S}^{(m)}}{\eta_{0}}=\frac{P_{1}^{(m)}(\xi)}{40 \pi} \int_{0}^{\infty} d y \frac{y^{2}}{1+\phi_{T} h_{H S}^{d,(m)}(y ; \xi)} \frac{\left(S_{H S}(y)^{\prime}\right)^{2}}{S_{H S}(y)}
$$

and

$$
\frac{\Delta \eta_{E L}^{(m)}}{\eta_{0}}=\frac{P_{1}^{(m)}(\xi)}{40 \pi} \int_{0}^{\infty} d y \frac{y^{2}}{1+\phi_{T} \overline{z^{2}} h_{E L}^{d,(m)}(y ; \xi)} \frac{\left(S_{E L}(y)^{\prime}\right)^{2}}{S_{E L}(y)} .
$$

Equations (104) and (105) generalize the MCT-HIs viscosity expressions we have derived before ${ }^{30}$ for dipolar farfield HIs and stick BC, to the more general case of mixed slipstick BCs. If the HIs in the dynamic input matrix, $\mathbf{F}^{S}(q, t)$, to the MCT shear relaxation function $\Delta \eta(t)$ of form discussed in our previous work, ${ }^{30}$ are treated on the PA level of approximation with stick BC, the denominator, $1+\phi_{T} h_{Y}^{d,(m)}(y)$, in Eqs. (104) and (105) must be replaced by $H_{N N}(y) / D^{0}$ for $Y$ $=H S$, and $H_{Z Z}(y) / D^{0}$ for $Y=E L$, using $D^{0}=D^{0}(\xi=0)$.

In Fig. 9, the simplified MCT-HIs predictions by Eqs. (99)-(105) for the relative excess viscosity, $\left(\eta-\eta_{0}\right) / \eta_{0}$, of an aqueous symmetric 1:1 electrolyte are compared with experimental data ${ }^{66}$ for $\mathrm{NaCl}$ in water at $25^{\circ} \mathrm{C} .{ }^{67}$ In Fig. 9(a), the MCT-HIs results have been obtained using $\sigma=2.82 \AA$, which is the (mean) Pauling ion diameter value employed by Chandra and $\mathrm{Bagchi}^{28}$ in their MCT-DDFT viscosity result represented by the green thick line segment. In both theoretical methods, the Attard PDFs of equal-sized ions have been used. However, different from our MCT-HIs approach, HIs are disregarded in the MCT-DDFT approach both in the shear relaxation and high-frequency viscosity contributions. Thus, $\Delta \eta_{\infty}$ is disregarded in particular. However, as we have shown in Ref. 30, $\Delta \eta_{\infty}$ contributes in fact strongly to $\eta$.

The theoretical predictions for $\Delta \eta_{e x c}$ in Fig. 9(a) by both methods severely underestimate the experimental data. This underestimation is more pronounced for the MCT-DFFT method. Chandra and $\mathrm{Bagchi}^{28}$ attribute this to the disregarded ion-specific effects in their approach. However, according to our simplified MCT-HIs analysis where $\Delta \eta_{\infty}$ and the influence of the HIs on $\Delta \eta$ are accounted for, the experimental viscosity is in fact well reproduced for stick BC provided that instead of $\sigma=2.82 \AA$, the physically meaningful mean diameter $\sigma=4.58 \AA$ of the hydrated $\mathrm{Na}^{+}$and $\mathrm{Cl}^{-}$ions in water is used (see Fig. 9(b)). Using the PA-HIs approximation instead of the RP-HIs approximation in the relaxation contributions, leads to a small viscosity increase only. The experimental viscosity data are underestimated when the non-zero slip parameter $\xi=1 / 7$, corresponding to $l_{p}=a / 4$, is used. The viscosity is thus seen to be more sensitive to changes in the hydrodynamic surface slip than the molar conductivity. 

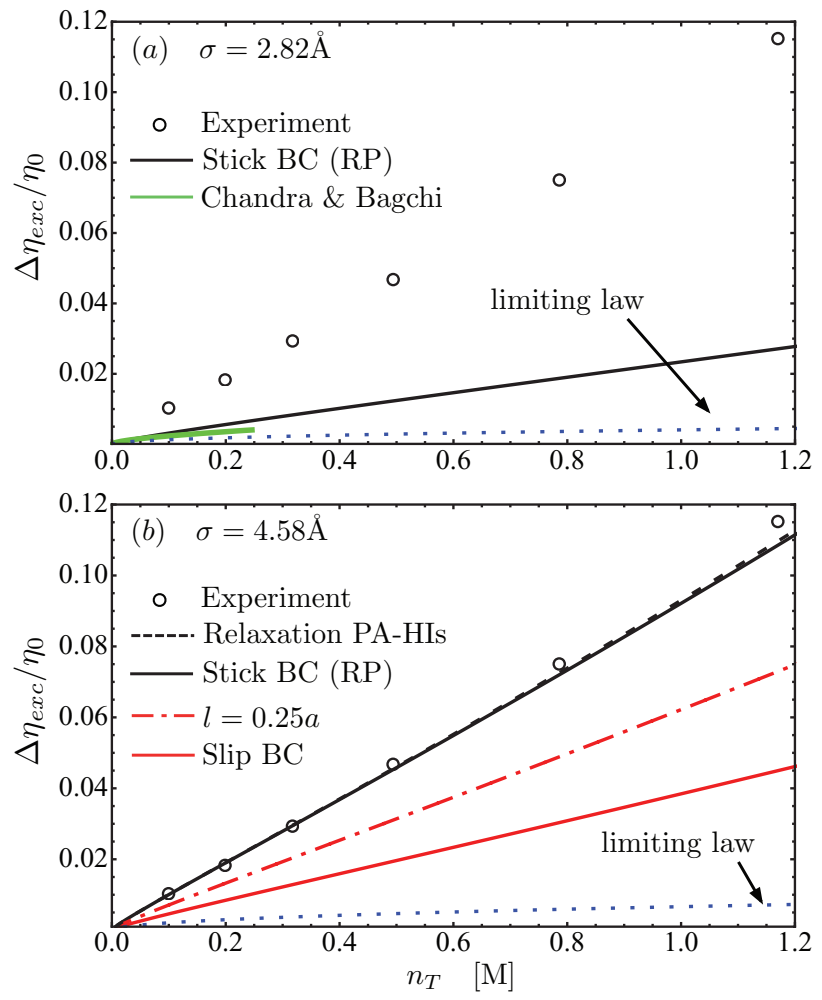

FIG. 9. Excess viscosity, $\Delta \eta_{e x c}$, in units of the solvent viscosity $\eta_{0}$, as function of the total ion concentration. Experimental data (open circles) for $\mathrm{NaCl}$ in water at $T=25^{\circ} \mathrm{C}^{66}$ are compared to theoretical predictions for an aqueous 1:1 electrolyte consisting of equal-sized cations and anions. (a) Simplified MCT-HIs (black solid line) and MCT-DDFT (green line segment) viscosity results. ${ }^{28}$ The Attard PDFs for $\sigma=2.82 \AA$ are used in both methods In the MCT-HIs result, the RP-HIs description is used for $\Delta \eta$, and Eq. (98) for $\Delta \eta_{\infty}$. (b) MCT-HIs viscosity results as in (a), but now using the (mean) ion diameter $\sigma=4.58 \AA$ consistent with the employed PM description. Solid black (red) line: Excess viscosity using dipolar far-field HIs and stick (perfect slip) BC for the shear relaxation part $\Delta \eta$, and Eq. (98) (Eqs. (99)-(101)) for $\Delta \eta_{\infty}$. Dashed black line: MCT-HIs result using PA-HIs for $\Delta \eta$. Dasheddotted red line: MCT-HIs result for mixed slip-stick spheres of slip length $l_{\text {slip }}=a / 4$, using dipolar HIs for $\Delta \eta$ and Eqs. (99)-(101) for $\Delta \eta_{\infty}$. Shown is also the limiting law result (dotted curve). ${ }^{30}$

\section{SUMMARY AND CONCLUSIONS}

On the basis of a simplified treatment of the multicomponent MCT method for electrolyte solutions with HIs developed in Paper I ${ }^{85}$ we have derived semi-analytic expressions for linear conduction-diffusion properties of strong electrolyte solutions consisting of equal-sized ions. The ions are treated on the PM-Smoluchowski dynamics level as charged Brownian spheres embedded in a Newtonian continuous fluid. The simplified MCT-HIs conduction-diffusion and viscosity expressions evaluated in this work can be straightforwardly implemented on a personal computer, allowing for a fast calculation of transport properties over a vast parameter range. The numerical simplicity of the simplified MCT-HIs method makes it useful also to experimentalists. An important advantage of the method is that one can study individually the effects of the HIs on the short-time and relaxation parts of the transport coefficients, for different hydrodynamic BCs and ion-sphere hydrodynamic models. In the point-ion limit, the conduction-diffusion transport coefficients with HIs as derived in Paper I ${ }^{85}$ are recovered. For very low ion concen- trations where HIs have no influence on the ion cloud relaxation, the DFOF low-concentration limiting law expressions for conduction-diffusion coefficients and the viscosity are reproduced.

The only required input are the equilibrium PDFs of the PM. The Attard PDFs for monovalent ions have been used throughout for analytic simplicity. We have checked that transport coefficient results based on the more elaborate MSA PDFs are numerically close to those based on the Attard input, up to the largest considered concentration.

We have shown that both the short-time and the relaxation part of the transport coefficients are considerably influenced by the HIs. The MCT-HIs results for stick BC, and HIs treated on the RP dipolar far-field level, have been compared to results based on the PA approximation. Provided the average diameter $\sigma=4.58 \AA$ of hydrated $\mathrm{Na}^{+}$and $\mathrm{Cl}^{-}$ions in water is used, consistent with the underlying continuum mechanics picture of the solvent, the experimental values for the molar conductivity and viscosity of aqueous $\mathrm{NaCl}$ solutions are overall well reproduced for stick hydrodynamic surface boundary condition, without any adjustable parameter. If one allows for some hydrodynamic slip (i.e., $\xi=1 / 7$ corresponding to $l_{\text {slip }}=a / 4$ ), the agreement between the MCT-HIs predicted molar conductivity and the experimental data becomes even excellent. This finding is consistent with the existence of ion hydration layers expected to cause some hydrodynamic slip, by an amount depending on the molecular structure and width of the hydration shell, and for thicker shells also on the form of the local flow field near the ions.

A key observation in this study is that the invocation of RP-HIs with stick BC in the simplified MCT-HIs method leads to results which are in consistently good overall agreement with the experimentally determined collective transport properties of aqueous $\mathrm{NaCl}$ solutions. This observation is not fortuitous since for small electrolyte ions, and in the considered volume fraction range, the far-field HIs part is expected to contribute most strongly. Lubrication interactions, which are included in the more elaborate PA treatment of the HIs, are likely to be of no relevance for the nano-sized (hydrated) ions, different from colloidal particles which are typically several hundred nanometers in size. Moreover, the PA-based treatment of the HIs tends to overestimate the slowing effect of near-field HIs on the ion conductivity and electrophoretic mobilities, and on the (short-time) self-diffusion and sedimentation coefficients.

The simplified MCT-HIs method presented in a selfcontained way in Paper $\mathrm{I}^{85}$ and in the present paper, can be extended in several ways some of which we are currently exploring. We are in the process of studying the influence of HIs on the chemical (mutual) diffusion of electrolyte ions in weak concentration gradients (see also Refs. 70-73), and the performance of generalized Stokes-Einstein and NernstEinstein relations between conduction-diffusion properties and the electrolyte viscosity. Moreover, we are currently extending the MCT-HIs method to size-asymmetric electrolytes, and electrolytes including non-monovalent ion species. These extensions require improved PM pair correlation functions as input which comply with the Stillinger-Lovett moment conditions, ${ }^{74,75}$ and account in addition for ion-pairing and 
nonlinear screening tendencies. For this purpose, MC computer simulations of the PM PDFs based on the Ewald ${ }^{76}$ and Wolf ${ }^{77}$ methods are under way. ${ }^{78}$ These simulation tools can be applied also to strongly asymmetric electrolytes. ${ }^{79,80}$

Note that for asymmetric electrolytes, the convenient orthogonality properties in Eqs. (8) and (9), which are specific to the linear MSA and Attard PDFs of equal-sized ions, do not apply any more. Consequently, additional cross contributions to the transport coefficients must be considered. These contributions invoke in particular the global dynamic structure factor $F_{N Z}(q, t)$, describing dynamic cross correlations between fluctuations in the total number and charge densities (cf. Paper $\mathrm{I}^{30,85}$ ). If large ion concentrations are considered, a self-consistent extension of the simplified MCT-HIs can be developed as outlined in Paper I, ${ }^{85}$ which accounts for HIs also in the MCT vertex function contributions to the collective memory function matrix. Furthermore, the MCT-HIs approach can be generalized, with some effort, to frequencydependent transport properties.

Finally, the MCT-HIs method can be generalized to strongly size and charge asymmetric polyion solutions where the polyions can be treated as a colloidal macroion species. ${ }^{81}$ Examples in case are salty solutions of globular proteins such as apoferritin ${ }^{82}$ and bovine serum albumin. ${ }^{83}$ For these systems, a first-order expansion of the macroion transport coefficients in terms of the small ratio of macroion and salt ion diffusion coefficients is quite useful. Such an expansion was used by McPhie and Nägele ${ }^{84}$ in their study of the ion cloud relaxation effect on the long-time self- and tracer diffusion coefficients of charged colloidal particles. Work in this direction, but now for collective transport properties, is in progress.

\section{ACKNOWLEDGMENTS}

This work is supported by the Deutsche Forschungsgemeinschaft (DFG) (SFB-TR6, Project No. B2). We thank Marco Heinen (University of Düsseldorf) and Mathieu McPhie (GeorgeTech-Lorraine University) for helpful discussions, and Bogdan Cichocki (Warsaw University) for having brought Ref. 65 to our attention.

\section{APPENDIX A: HYDRODYNAMIC FUNCTIONS OF EQUAL-SIZE ION SOLUTIONS}

The short-time self-diffusion coefficients and distinct partial hydrodynamic functions, for HIs treated on the PA level, follow from inserting Eq. (22) into Eqs. (I.36) and (I.37). The result is

$$
\frac{D_{\alpha}^{S}}{D^{0}}=1+8 \phi_{T} \int_{1}^{\infty} d x x^{2}\left(\sum_{\gamma=1}^{m} x_{\gamma} g_{\alpha \gamma}(x)\right) K^{S, N F}(x),
$$

and

$$
H_{\alpha \beta}^{d}(y)=H_{\alpha \beta}^{d, R P}(y)+H_{\alpha \beta}^{d, N F},
$$

where in Eq. (A1) we have identified the hydrodynamic radius with the excluded volume radius of the equal-sized ions. The distinct partial hydrodynamic function is the sum of the RP far-field part,

$$
\begin{aligned}
\frac{H_{\alpha \beta}^{d, R P}(y)}{D^{0}}= & -15 \phi_{T}\left(x_{\alpha} x_{\beta}\right)^{1 / 2} \frac{j_{1}(y)}{y} \\
& +18 \phi_{T}\left(x_{\alpha} x_{\beta}\right)^{1 / 2} \int_{1}^{\infty} d x x K^{d, R P}(y, x) h_{\alpha \beta}(x)
\end{aligned}
$$

and the near-field part

$$
\frac{H_{\alpha \beta}^{d, N F}(y)}{D^{0}}=24 \phi_{T}\left(x_{\alpha} x_{\beta}\right)^{1 / 2} \int_{1}^{\infty} d x x^{2} K^{d, N F}(y, x) g_{\alpha \beta}(x) .
$$

We have introduced here the self- and distinct hydrodynamic kernel functions for stick BC, given by

$$
\begin{gathered}
K^{S, N F}(x)=x_{11}^{N F}(x)+2 y_{11}^{N F}(x), \\
K^{d, R P}(y, x)=j_{0}(y x)-\frac{j_{1}(y x)}{y x}+\frac{j_{2}(y x)}{6 x^{2}},
\end{gathered}
$$

and

$$
\begin{aligned}
K^{d, N F}(y, x)= & y_{12}^{N F}(x) j_{0}(y x) \\
& +\left[x_{12}^{N F}(x)-y_{12}^{N F}(x)\right]\left[\frac{j_{1}(y x)}{y x}-j_{2}(y x)\right] .
\end{aligned}
$$

For the MSA and Attard static inputs to the $m$-component PM of equal-sized ions where Eq. (15) holds sway, total electroneutrality implies that the short-time self-diffusion coefficients, $D_{\alpha}^{S}$, of all $m$ ion components are equal to the short-time self-diffusion coefficient, $D_{H S}^{S}$, of a monodisperse system of neutral hard spheres with volume fraction $\phi_{T}$, independent of the component index. The neutral hard-sphere short-time self diffusion coefficient for stick hydrodynamic BC and PA treatment of HIs is given by ${ }^{39,40}$

$$
\begin{aligned}
\frac{D_{H S}^{S}}{D^{0}} & =1+8 \phi_{T} \int_{1}^{\infty} x^{2} g_{H S}(x) K^{S, N F}(x) d x \\
& =1-1.8315 \phi_{T}+\mathcal{O}\left(\phi_{T}^{2}\right),
\end{aligned}
$$

where $g_{H S}(x)=1+h_{H S}(x)$ is the equilibrium PDF of neutral hard spheres, and $h_{H S}(x)$ is the corresponding total correlation function. The numerically precise second-order virial part, $-0.219 \phi^{2}$, in $D_{H S}^{S} / D^{0}$ invokes additional three-body HIs contributions and has been determined by Cichocki et al. ${ }^{41}$ As shown before ${ }^{42}$ by comparison with Stokesian dynamics simulations, the PA-approximation-based $D_{H S}^{S}$ underestimates the simulation data for the hard-sphere self-diffusion coefficient for volume fractions $\phi \gtrsim 0.04$.

Thus, for an electrolyte mixture of equal-sized ions, one obtains $D_{\alpha}^{S}=D^{S}=D_{H S}^{S}$ with

$$
\frac{D^{S}}{D^{0}}=1+\phi_{T} \mathrm{~h}_{H S}^{S, N F}
$$

in PA approximation, where

$$
\mathrm{h}_{H S}^{S, N F}=8 \int_{1}^{\infty} d x x^{2} g_{H S}(x) K^{S, N F}(x) .
$$


That all ion components share the same short-time selfdiffusion coefficient equal to the hard-sphere coefficient, even in the case of ions with different valence, is a consequence of the special decomposition property in Eq. (15) which is obeyed by the linear Attard and MSA PDFs. These PDFs are a decently good description for monovalent ions only. Note here that for a binary symmetric $z: z$ electrolyte of cations of concentration $n_{+}=n_{T} / 2$ and valency $z_{+}=z \geq 1$, and anions of concentration $n_{-}=n_{+}$and valency $z_{-}=-z$, the symmetry of the system implies the equality of the short-time $\left(D_{\alpha}^{S}=D^{S}\right)$ and long-time $\left(D_{\alpha}^{L}=D^{L}\right)$ self-diffusion coefficients of cations and anions. Moreover, $\overline{z^{2}}=z^{2}$ and all odd valency moments are zero.

For an electrolyte mixture of equal-sized, hydrodynamically equivalent ions with static pair correlations described on the MSA or Attard levels of approximation, the $m \times m$ hydrodynamic function matrix decouples into hard-sphere and electro-steric parts according to

$$
\mathbf{H}(y)=D^{S} \mathbf{Q}+H_{N N}(y) \mathbf{P}_{n}+\frac{1}{\overline{z^{2}}} H_{Z Z}(y) \mathbf{P} .
$$

Here,

$$
H_{Z Z}(y)=\overline{z^{2}} \operatorname{Tr}[\mathbf{P} \cdot \mathbf{H}(y)]
$$

and

$$
H_{N N}(y)=\operatorname{Tr}\left[\mathbf{P}_{n} \cdot \mathbf{H}(y)\right],
$$

are the global number-number and charge-charge hydrodynamic functions associated, respectively, with short-time correlations in the total charge density (labeled by $Z$ ) and total number density fluctuations (labeled by $N$ ). The matrix trace operation is denoted by Tr. The two global hydrodynamic functions, for HIs treated on the PA level, are given explicitly by

$$
H_{N N}(y)=D^{S}+D^{0} \phi_{T}\left(\mathrm{~h}_{H S}^{d, R P}(y)+\mathrm{h}_{H S}^{d, N F}(y)\right)
$$

and

$$
H_{Z Z}(y)=\overline{z^{2}}\left[D^{S}+D^{0} \phi_{T} \overline{z^{2}}\left(\mathrm{~h}_{E L}^{d, R P}(y)+\mathrm{h}_{E L}^{d, N F}(y)\right)\right],
$$

respectively. We have introduced here the wavenumberdependent RP hard-sphere and electro-steric distinct constituent functions

$$
\begin{aligned}
\mathrm{h}_{H S}^{d, R P}(y) & =-15 \frac{j_{1}(y)}{y}+18 \int_{1}^{\infty} d x x K^{d, R P}(y, x) h_{H S}(x) \\
& =1+18 \int_{0}^{\infty} d x x K^{d, R P}(y, x) h_{H S}(x)
\end{aligned}
$$

and

$$
\mathrm{h}_{E L}^{d, R P}(y)=18 \int_{0}^{\infty} d x x K^{d, R P}(y, x) h_{E L}(x),
$$

respectively.

The related functions accounting for two-body near-field HIs are

$$
\mathrm{h}_{H S}^{d, N F}(y)=24 \int_{1}^{\infty} d x x^{2} K^{d, N F}(y, x) g_{H S}(x)
$$

and

$$
\mathrm{h}_{E L}^{d, N F}(y)=24 \int_{1}^{\infty} d x x^{2} K^{d, N F}(y, x) g_{E L}(x) .
$$

In the DH point-particle limit where $h_{H S}(r)=0$ and $h_{E L}(r)$ $=-L_{B} \exp \{-\kappa r\} / r$, the hydrodynamic function matrix in Eq. (A11) reduces to

$$
\mathbf{H}(q)=D^{0} \mathbf{1}-D_{\kappa} F^{O s}(q / \kappa) \mathbf{P} .
$$

Here, $D_{\kappa}$ is a self-diffusion coefficient associated with a spherical body of radius equal to the Debye length (cf. Eq. (I.84)). The Oseen function $F^{O_{s}}(u)$ is given in Eq. (I.83). The expression in Eq. (A20) for the matrix $\mathbf{H}(q)$ has been used in Paper $\mathrm{I}^{85}$ as input for the calculation of conductiondiffusion transport properties of hydrodynamically interacting point ions.

\section{APPENDIX B: SELF- AND CROSS-CORRELATION CONTRIBUTIONS TO $\Delta \mu^{L}$}

To identify the self-correlation and cross-correlation parts of $\Delta \boldsymbol{\mu}$, the terms in the inverse, $\mathbf{D}^{-1}$, of the matrix $\mathbf{D}=\mathbf{1}+\mathbf{H}^{-1} \cdot \mathbf{m}^{c, i r r}$ which are of zeroth-order in $n_{\alpha^{*}}$ need to be isolated. This is achieved using

$$
\mathbf{H}^{-1}=\frac{1}{D^{S}} \mathbf{1}-\frac{H_{N N}-D^{S}}{H_{N N} D^{S}} \mathbf{P}_{n}-\frac{H_{Z Z}-\overline{z^{2}} D^{S}}{H_{Z Z} D^{S}} \mathbf{P}
$$

in combination with the splitting,

$$
\mathbf{m}^{c, i r r}=\left(\mathbf{m}^{c, i r r}\right)^{\text {self }}+\left(\mathbf{m}^{c, i r r}\right)^{\text {cross }},
$$

of $\mathbf{m}^{c, i r r}$ into its self-correlation and cross-correlation parts. Here, $\left(\mathbf{m}^{c, i r r}\right)^{\text {self }}$ is a diagonal matrix. In writing $\mathbf{D}=\mathbf{A}+\mathbf{B}$, we get

$$
\mathbf{A}=\mathbf{1}+\frac{1}{D^{S}}\left(\mathbf{m}^{c, i r r}\right)^{\text {self }}
$$

for the diagonal matrix $\mathbf{A}$, and

$$
\begin{aligned}
\mathbf{B}= & \frac{1}{D^{S}}\left(\mathbf{m}^{c, i r r}\right)^{\text {cross }} \\
& -\left(\frac{H_{N N}-D^{S}}{H_{N N} D^{S}} \mathbf{P}_{n}-\frac{H_{Z Z}-\overline{z^{2}} D^{S}}{H_{Z Z} D^{S}} \mathbf{P}\right) \cdot \mathbf{m}^{c, i r r}
\end{aligned}
$$

for the remainder. Next, we use the matrix identity,

$$
(\mathbf{A}+\mathbf{B})^{-1}=\mathbf{A}^{-1}-\left[\mathbf{A}^{-1} \cdot \mathbf{B} \cdot(\mathbf{A}+\mathbf{B})^{-1}\right]_{S},
$$

where $[\cdots]_{S}$ means that the symmetric matrix part is taken. Eq. (B5) follows from the Woodbury matrix identity ${ }^{47}$ in combination with a Searle matrix identity. The latter identity reads $^{48}$

$$
\left(\mathbf{A}^{-1}+\mathbf{B}^{-1}\right)^{-1}=\mathbf{A} \cdot(\mathbf{A}+\mathbf{B})^{-1} \cdot \mathbf{B}=\mathbf{B} \cdot(\mathbf{A}+\mathbf{B})^{-1} \cdot \mathbf{A} .
$$

In this way, we obtain the diagonal self-correlation matrix part of the relaxation mobility matrix as

$$
k_{B} T(\Delta \boldsymbol{\mu})^{\text {self }}=-\left(\mathbf{m}^{c, i r r}\right)^{\text {self }} \cdot \mathbf{A}^{-1},
$$


implying

$$
k_{B} T\left(\mu_{\alpha \alpha}\right)^{\text {self }}=D_{\alpha}^{L},
$$

for the self-correlation matrix elements of the steady-state mobility matrix. For the cross-correlation part of the relaxation mobility matrix, the more involved matrix expression

$$
\begin{aligned}
(\Delta \boldsymbol{\mu})^{\text {cross }}= & -\left[\left(\mathbf{m}^{c, i r r}\right)^{\text {cross }} \cdot \mathbf{A}^{-1}\right]_{S} \\
& +\left[\mathbf{m}^{c, i r r} \cdot\left[(\mathbf{A}+\mathbf{B})^{-1} \cdot \mathbf{B} \cdot \mathbf{A}^{-1}\right]_{S}\right]_{S}
\end{aligned}
$$

is obtained.

\section{APPENDIX C: SEDIMENTATION COEFFICIENT FOR THE MIXED SLIP-STICK AND PERMEABLE-SPHERE MODELS}

An analysis similar to that in Sec. IV for ions with mixed slip-stick BCs can be made for solvent-permeable PM ions. We restrict our analysis in the following to the sedimentation coefficient of permeable ions, by discussing in addition the effect of mixed slip-stick BCs. While not directly measurable for electrolyte solutions, the sedimentation coefficient contributes, according to Eqs. (A11) and (A13), to the mobility matrix $\boldsymbol{\mu}^{L}$.

We recall that for equal-sized ions and Attard or MSA static PDFs, the ion sedimentation coefficient is equal to the sedimentation coefficient, $K_{H S}^{s e d}=H_{N N} / D^{0}$, of a homogeneous dispersion of monodisperse hard spheres. A closed analytic expression for the sedimentation coefficient, $K_{H S}^{\text {sed, }(m)}$, of a concentrated dispersion of mixed slip-stick hard spheres, with HIs treated on the dipolar far-field level, is obtained from using the analytic PY solution ${ }^{24,31}$ for $g_{H S}(x)$ in the evaluation of $\mathrm{h}_{H S}^{d,(m)}(y \rightarrow 0 ; \xi)$. The PY- $g_{H S}(x)$ is quite accurate for volume fractions $\phi_{T} \lesssim 0.4$. In this way, we obtain

$$
\mathrm{h}_{H S}^{d,(m)}(y \rightarrow 0 ; \xi)=P_{2}(\xi)-\frac{3}{5} P_{1}(\xi) \frac{\phi_{T}^{2}-2 \phi_{T}+10}{2 \phi_{T}+1}
$$

for the zero-wavenumber form of the (reduced) distinct hydrodynamic function part. Using Eq. (A14), this results in the analytic expression,

$$
\begin{aligned}
K_{H S}^{\text {sed },(m)} & =1+P_{2}(\xi) \phi_{T}-\frac{3}{5} P_{1}(\xi) \frac{\phi_{T}^{2}-2 \phi_{T}+10}{1+2 \phi_{T}} \phi_{T} \\
& =1+\left(P_{2}(\xi)-6 P_{1}(\xi)\right) \phi_{T}+\frac{66}{5} P_{1}(\xi) \phi_{T}^{2}+\mathcal{O}\left(\phi_{T}^{3}\right)
\end{aligned}
$$

for the sedimentation coefficient of a homogeneous dispersion of mixed slip-stick hard spheres. For stick BC, this expression reduces to the result,

$$
\begin{aligned}
K_{H S}^{\text {sed,stick }} & =\frac{\left(1-\phi_{T}\right)^{3}}{1+2 \phi_{T}}+\frac{\phi_{T}^{2}}{5} \\
& =1-5 \phi_{T}+\frac{66}{5} \phi_{T}^{2}+\mathcal{O}\left(\phi_{T}^{3}\right),
\end{aligned}
$$

derived earlier by Banchio and Nägele. ${ }^{40}$ The additive contribution, $\phi_{T}^{2} / 5$, is significant at larger volume fractions, and it was left out by Brady and Durlofski ${ }^{69}$ in their derivation of

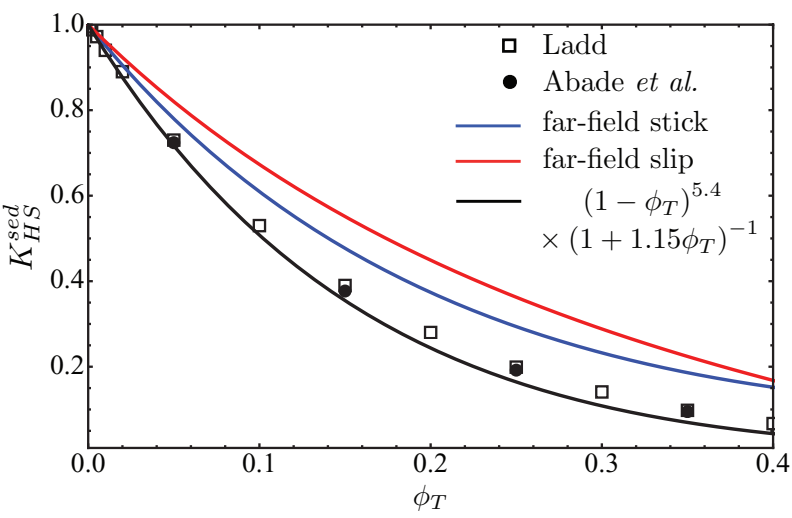

FIG. 10. Sedimentation coefficient of impermeable neutral hard spheres as a function of the total volume fraction. The analytic result in Eq. (C2), based on the dipolar HIs approximation and the Percus-Yevick static input, is compared with simulation data by Abade et al. (black filled circles) ${ }^{60}$ and Ladd (black open squares) ${ }^{68}$ for hydrodynamically stick spheres. Blue and red solid lines: Theoretical predictions for stick and perfect slip spheres according to Eqs. (C3) and (C4), respectively. Black solid line: Experimental fit curve according to Eq. (C5).

$K_{H S}^{\text {sed,stick }}$ based on the RP approximation and the PY static input. Equation (C3) has been re-derived recently by Gilleland et al. using a variational method. ${ }^{38}$

For perfect slip BC, Eq. (C2) specializes to

$$
\begin{aligned}
K_{H S}^{\text {sed,slip }} & =1-\frac{2}{5} \frac{\phi_{T}^{2}-2 \phi_{T}+10}{1+2 \phi_{T}} \phi_{T} \\
& =1-4 \phi_{T}+\frac{44}{5} \phi_{T}^{2}+\mathcal{O}\left(\phi_{T}^{3}\right) .
\end{aligned}
$$

Figure 10 displays the volume fraction dependence of the sedimentation coefficient of hard spheres, as predicted by Eq. (C3) for stick, and Eq. (C4) for perfect slip BCs. The analytic results are compared with simulation results, ${ }^{60,68}$ and with the fit formula by Gilleland et al., ${ }^{38}$

$$
K_{H S}^{\text {sed,stick }}=\frac{\left(1-\phi_{T}\right)^{5.4}}{1+1.15 \phi_{T}} .
$$

This fit formula constitutes an overall representation of experimental sedimentation data for hydrodynamically stick colloidal hard spheres.

Numerically precise first-order virial expansion results for the hard-sphere sedimentation coefficient have been obtained by Cichocki and Felderhof. ${ }^{65}$ Their result is

$$
K_{H S}^{\text {sed,stick }}=1-6.546 \phi_{T}+\mathcal{O}\left(\phi_{T}^{2}\right)
$$

for the stick BC, and

$$
K_{H S}^{\text {sed,slip }}=1-4.489 \phi_{T}+\mathcal{O}\left(\phi_{T}^{2}\right) .
$$

for the perfect slip BC. Thus, Eq. (C5) has built into it the correct first-order virial term for hydrodynamically stick spheres. The first-order in concentration coefficients in the analytic expressions in Eqs. (C3) and (C4) for stick and perfect slip BCs, respectively, underestimate to some extent the magnitudes of the numerically precise first-order virial coefficients.

According to Fig. 10, the simulation data are decently well described by the analytic expression for $K_{H S}^{\text {sed,stick }}$ in Eq. (C3) for $\phi_{T} \lesssim 0.1$, but at larger $\phi_{T}$ the simulation data 


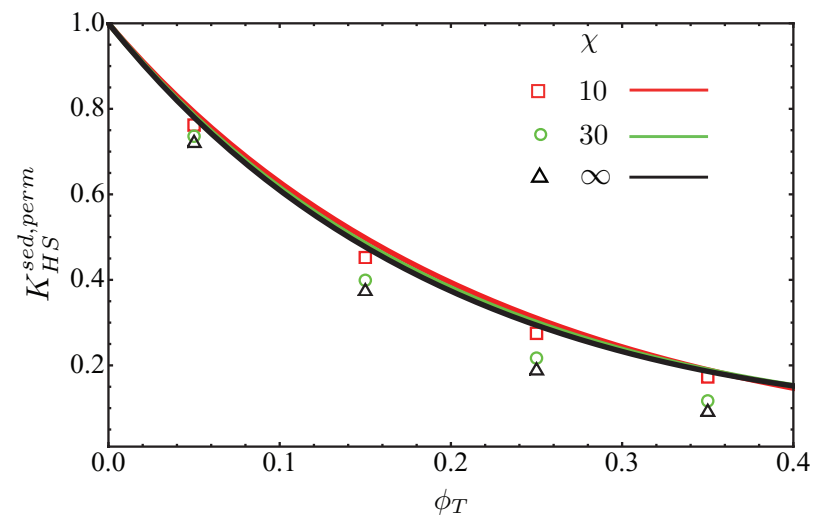

FIG. 11. Short-time sedimentation coefficient, $K_{H S}^{\text {sed, perm }}$, of uniformly permeable hard spheres. Symbols are simulation results by Abade et al., ${ }^{60}$ for values of the inverse reduced penetration depth $\chi$ as indicated. For $\chi=10$, e.g., the penetration depth is one tenth of the particle radius. Solid lines are analytical predictions according to Eq. (C2) wherein $P_{i}^{(p)}(\chi)$ is substituted for $P_{i}^{(m)}(\xi)$, for $i=1,2$.

are significantly overestimated. This can be attributed to nearfield many-body HIs contributions neglected in Eq. (C3). If the PA approximation is used instead in calculating the sedimentation coefficient, the influence of the near-field HIs is overestimated, with nonphysical negative values of $K_{H S}^{\text {sed,stick }}$ predicted $^{39}$ for $\phi_{T}>0.22$.

The sedimentation coefficient of perfect slip BC hard spheres is in general larger than that for stick BC spheres, owing to the reduced hydrodynamic coupling of the particles.

An analytic expression for the sedimentation coefficient, $K_{H S}^{\text {sed,perm }}$, of uniformly permeable hard spheres with PY static input and in dipolar HIs approximation follows readily from Eq. (C2) by replacing $P_{i}^{(m)}(\xi)$ with $P_{i}^{(p)}(\chi)$ for $i=1,2$. For impermeable spheres $\chi=\infty$, and Eq. (C3) for stick BC spheres is recovered from this analytic expression. Figure 11 shows the sedimentation coefficient of uniformly permeable spheres predicted by the analytic expression, for three values of $\chi$ as indicated. The analytic predictions are compared with hydrodynamic force multipole simulation data by Abade et $a l .{ }^{60}$ The simulation data are overestimated for larger volume fractions. For $\phi_{T} \lesssim 0.35$, the increase of the sedimentation coefficient with increasing permeability (decreasing $\chi$ ) is qualitatively correctly predicted but underestimated quantitatively.

We close this appendix on noting that the ion sedimentation coefficient contributes to the chemical (mutual) diffusion coefficient of a binary electrolyte. The latter coefficient can be determined experimentally. Chemical diffusion will be analyzed, using the MCT-HIs method, in a forthcoming publication.

${ }^{1}$ A. Anderko, P. Wang, and P. Rafal, Fluid Phase Equilib. 194-197, 123 (2002).

${ }^{2}$ J. M. G. Barthel, H. Krienke, and W. Kunz, Physical Chemistry of Electrolyte Solutions, Topics in Physical Chemistry, Vol. 5 (Steinkopff, Darmstadt, 1998).

${ }^{3} \mathrm{H}$. Falkenhagen and W. Ebeling, in Theorie der Elektrolyte, edited by S. Hirzel (Verlag, Stuttgart, 1971).

${ }^{4}$ H. Falkenhagen and M. Dole, Phys. Z 30, 611 (1929).

${ }^{5}$ L. Onsager and R. M. Fuoss, J. Phys. Chem. 36, 2689 (1932).
${ }^{6}$ H. Falkenhagen and E. L. Vernon, Philos. Mag. Series 7 74, 537 (1932).

${ }^{7}$ L. Onsager, Ann. N.Y. Acad. Sci. 46, 241 (1945).

${ }^{8}$ L. Onsager and S. K. Kim, J. Phys. Chem. 61, 215 (1957).

${ }^{9}$ J. Hubbard and L. Onsager, J. Chem. Phys. 67, 4850 (1977).

${ }^{10}$ J. Hubbard and P. Wolynes, J. Chem. Phys. 69, 998 (1978).

${ }^{11}$ B. Bagchi, J. Chem. Phys. 109, 3989 (1998).

${ }^{12}$ P. Attard, Phys. Rev. E 48, 3604 (1993).

${ }^{13}$ J. P. Hansen and I. R. Mc Donald, Theory of Simple Liquids, 3rd ed. (Elsevier, Amsterdam, 2006).

${ }^{14}$ G. Nägele, Phys. Rep. 272, 215 (1996).

${ }^{15}$ J.-F. Dufreche, O. Bernard, and P. Turq, J. Mol. Liq. 118, 189 (2005).

${ }^{16}$ W. Ebeling, R. Feistel, G. Kelbg, and R. Sandig, J. Non-Equilib. Thermodyn. 3, 11 (1978).

${ }^{17}$ W. Ebeling and J. Rose, J. Solution Chem. 10, 599 (1981).

${ }^{18}$ O. Bernard, W. Kunz, P. Turq, and L. Blum, J. Phys. Chem. 96, 3833 (1992).

${ }^{19}$ O. Bernard, W. Kunz, P. Turq, and L. Blum, J. Phys. Chem. 96, 398 (1992).

${ }^{20}$ S. Durand-Vidal, P. Turq, O. Bernard, C. Treiner, and L. Blum, Physica A 231, 123 (1996).

${ }^{21}$ S. Durand-Vidal, P. Turq, and O. Bernard, J. Phys. Chem. 100, 17345 (1996).

${ }^{22}$ J. F. Dufreche, O. Bernard, S. Durand-Vidal, and P. Turq, J. Phys. Chem. B 109, 9873 (2005).

${ }^{23}$ G. M. Roger, S. Durand-Vidal, O. Bernard, and P. Turq, J. Phys. Chem. B 113, 8670 (2009).

${ }^{24}$ L. Blum and J. S. Høye, J. Phys. Chem. 81, 1311 (1977).

${ }^{25}$ K. Hiroike, Mol. Phys. 33, 1195 (1977).

${ }^{26}$ A. Chandra, R. Biswas, and B. Bagchi, J. Am. Chem. Soc. 121, 4082 (1999).

${ }^{27}$ A. Chandra and B. Bagchi, J. Chem. Phys. 110, 10024 (1999).

${ }^{28}$ A. Chandra and B. Bagchi, J. Phys. Chem. B 104, 9067 (2000).

${ }^{29}$ A. Chandra and B. Bagchi, J. Chem. Phys. 113, 3226 (2000).

${ }^{30}$ C. Contreras-Aburto and G. Nägele, J. Phys.: Condens. Matter 24, 464108 (2012).

${ }^{31}$ M. S. Wertheim, Phys. Rev. Lett. 10, 321 (1963).

${ }^{32}$ E. Waisman and J. L. Lebowitz, J. Chem. Phys. 56, 3093 (1972).

${ }^{33}$ J. K. G. Dhont, An Introduction to Dynamics of Colloids (Elsevier, Amsterdam, 1996), Vol. 2.

${ }^{34}$ S. Kim and S. Karilla, Microhydrodynamics (Butterworth-Heinemann, Boston, 1991).

${ }^{35}$ R. B. Jones and G. S. Burfield, Physica A 111, 562 (1982).

${ }^{36}$ D. J. Jeffrey and Y. Onishi, J. Fluid Mech. 139, 261 (1984).

${ }^{37}$ J. Rotne and S. Prager, J. Chem. Phys. 50, 4831 (1969).

${ }^{38}$ W. T. Gilleland, S. Torquato, and W. B. Russel, J. Fluid Mech. 667, 403 (2011).

${ }^{39}$ M. Heinen, A. J. Banchio, and G. Nägele, J. Chem. Phys. 135, 154504 (2011).

${ }^{40}$ A. J. Banchio and G. Nägele, J. Chem. Phys. 128, 104903 (2008).

${ }^{41}$ B. Cichocki, M. Ekiel-Jezewska, and E. Wajnryb, J. Chem. Phys. 111, 3265 (1999).

${ }^{42}$ M. Heinen, P. Holmqvist, A. J. Banchio, and G. Nägele, J. Appl. Crystallogr. 43, 970 (2010).

${ }^{43}$ E. L. Cussler, Diffusion: Mass Transfer in Fluid Systems, 2nd ed. (Cambridge University Press, Cambridge, 1997).

${ }^{44}$ G. Nägele, J. Bergenholtz, and J. K. G. Dhont, J. Chem. Phys. 110, 7037 (1999).

${ }^{45}$ G. Nägele and J. K. G. Dhont, J. Chem. Phys. 108, 9566 (1998).

${ }^{46}$ R. Mills and V. Lobo, Self-diffusion in Electrolyte Solutions (Elsevier, Amsterdam, 1989).

${ }^{47}$ G. H. Golub and C. F. Van Loan, Matrix Computations, 3rd ed. (Johns Hopkins University Press, Baltimore, 1996).

${ }^{48}$ S. R. Searle, Matrix Algebra Useful for Statistics, Wiley Series in Probability and Statistics (Wiley, 2006).

${ }^{49}$ P. Szymczak and B. Chichocki, Europhys. Lett. 59, 465 (2002).

${ }^{50}$ E. Wajnryb, P. Szymczak, and B. Cichocki, Physica A 335, 339 (2004).

${ }^{51}$ M. Jardat, O. Bernard, P. Turq, and G. R. Kneller, J. Chem. Phys. 110, 7993 (1999).

${ }^{52}$ D. G. Miller, J. Phys. Chem. 70, 2639 (1966).

${ }^{53}$ P. Atkins and J. de Paula, Physical Chemistry, 7th ed. (Oxford University Press, 2002).

${ }^{54}$ B. U. Felderhof, Physica A 84, 569 (1976).

${ }^{55}$ R. B. Jones and R. Schmitz, Physica A 149, 373 (1988).

${ }^{56}$ B. Cichocki, B. Felderhof, and R. Schmitz, PCH, PhysicoChem. Hydrodyn. 10, 383 (1988). 
${ }^{57}$ P. Reuland, B. U. Felderhof, and R. B. Jones, Physica A 93, 465 (1978).

${ }^{58}$ M. U. Bäbler, J. Sefcik, M. Morbidelli, and J. Bałdyga, Phys. Fluids 18, 013302 (2006).

${ }^{59}$ R. B. Jones, Physica A 92, 571 (1978).

${ }^{60}$ G. C. Abade, B. Cichocki, M. L. Ekiel-Jezewska, G. Nägele, and E. Wajnryb, J. Chem. Phys. 132, 014503 (2010).

${ }^{61}$ G. C. Abade, B. Cichocki, M. L. Ekiel-Jezewska, G. Nägele, and E. Wajnryb, J. Chem. Phys. 133, 084906 (2010).

${ }^{62}$ G. C. Abade, B. Cichocki, M. L. Ekiel-Jezewska, G. Nägele, and E. Wajnryb, J. Chem. Phys. 136, 104902 (2012).

${ }^{63}$ J. Anderson, P. McKenzie, and R. Webber, Langmuir 7, 162 (1991).

${ }^{64}$ B. Cichocki, M. L. Ekiel-Jezewska, and E. Wajnryb, J. Chem. Phys. 119, 606 (2003).

${ }^{65}$ B. Cichocki and B. U. Felderhof, J. Chem. Phys. 89, 1049 (1988).

${ }^{66}$ D. Out and J. Los, J. Solution Chem. 9, 19 (1980).

${ }^{67}$ In Fig. 7 of our work on viscosity, ${ }^{30}$ the concentration values of the viscosity data for $\mathrm{NaCl}$ in water were mistakenly multiplied by a factor of two. However, all the theoretical results shown there are correct.

${ }^{68}$ A. J. C. Ladd, J. Chem. Phys. 93, 3484 (1990).

${ }^{69}$ J. Brady and L. Durlofski, Phys. Fluids 31, 717 (1988).

${ }^{70}$ J. F. Dufreche, O. Bernard, and P. Turq, J. Chem. Phys. 116, 2085 (2002).

${ }^{71}$ J. F. Dufreche, O. Bernard, M. Jardat, and P. Turq, J. Chem. Phys. 118, 8116 (2003).
${ }^{72}$ B. U. Felderhof, J. Chem. Phys. 118, 8114 (2003).

${ }^{73}$ G.-H. Gao, H.-B. Shi, and Y.-X. Yu, Fluid Phase Equilib. 256, 105 (2007).

${ }^{74}$ F. J. Stillinger and R. Lovett, J. Chem. Phys. 49, 1991 (1968).

${ }^{75}$ D. J. Mitchell, D. A. McQuarrie, A. Szabo, and J. Groeneveld, J. Stat. Phys. 17, 15 (1977).

${ }^{76}$ P. P. Ewald, Ann. Phys. (Berlin) 369, 253 (1921).

${ }^{77}$ D. Wolf, P. Keblinski, S. R. Phillpot, and J. Eggebrecht, J. Chem. Phys. 110, 8254 (1999).

${ }^{78}$ J. Falcón-González, R. Castañeda-Priego, and A. Gil-Villegas, personal communication (2012).

${ }^{79}$ V. Lobaskin and P. Linse, J. Chem. Phys. 109, 3530 (1998).

${ }^{80}$ V. Lobaskin and P. Linse, J. Chem. Phys. 111, 4300 (1999).

${ }^{81}$ G. Nägele, M. Heinen, A. J. Banchio, and C. Contreras-Aburto, "Electrokinetic and hydrodynamic properties of charged-particles systems: From small electrolyte ions to large colloids," Eur. Phys. J. E (to be published).

${ }^{82}$ J. Gapinski, A. Wilk, A. Patkowski, W. Häußler, A. J. Banchio, R. Pecora, and G. Nägele, J. Chem. Phys. 123, 054708 (2005).

${ }^{83}$ M. Heinen, F. Zanini, F. Roosen-Runge, D. Fedunova, F. Zhang, M. Hennig, T. Seydel, R. Schweins, M. Sztucki, M. Antalik et al., Soft Matter 8, 1404 (2012).

${ }^{84}$ M. G. McPhie and G. Nägele, J. Chem. Phys. 127, 034906 (2007).

${ }^{85}$ C. Contreras Aburto and G. Nägele, J. Chem. Phys. 139, 134109 (2013). 